\title{
Efficient Loading of Mano Mn Particles On Calcined Laterite Soil (Lt-nMn) For Higher Removal of As(III) Ions From Groundwater: Adsorption And Eco-Scale Analysis
}

\section{Roshan Prabhakar}

Indian Institute of Technology (Indian School of Mines): Indian Institute of Technology

\section{Somaparna Ghosh}

Indian Institute of Technology (Indian School of Mines): Indian Institute of Technology

Ali Malik

Indian Institute of Technology (Indian School of Mines): Indian Institute of Technology

Sukha Ranjan Samadder ( $\sim$ samadder@iitism.ac.in )

IIT (ISM) Dhanbad https://orcid.org/0000-0002-0037-7030

\section{Research Article}

Keywords: Adsorption, As(III) removal, Batch adsorber design, nano Manganese particle, and Laterite

Posted Date: August 24th, 2021

DOl: https://doi.org/10.21203/rs.3.rs-767410/v1

License: (c) (i) This work is licensed under a Creative Commons Attribution 4.0 International License.

Read Full License 


\title{
Efficient loading of nano Mn particles on calcined laterite soil (Lt-nMn) for higher removal of As(III) ions from groundwater: Adsorption and eco-scale analysis
}

\section{By}

\author{
Roshan Prabhakar ${ }^{a}$, Somaparna Ghosh ${ }^{b}$, Ali Malik ${ }^{c}$, and Sukha Ranjan Samadder ${ }^{\text {* }}$
}

\begin{abstract}
${ }^{a}$ Research Scholar, Department of Environmental Science \& Engineering, Indian Institute of Technology (Indian School of Mines), Dhanbad-826004, India. (Email: roshanbt30@gmail.com)

${ }^{b}$ M. Tech Scholar, Department of Environmental Science and Engineering, Indian Institute of Technology (Indian School of Mines), Dhanbad-826004, India.

${ }^{c}$ M. Tech Scholar, Department of Environmental Science and Engineering, Indian Institute of Technology (Indian School of Mines), Dhanbad-826004, India.

$d^{*}$ Associate Professor, Department of Environmental Science \& Engineering, Indian Institute of Technology (Indian School of Mines), Dhanbad-826004, India, (*Corresponding author: Email: samadder@iitism.ac.in; sukh_samadder@yahoo.co.in; Phone No: +919471191823; Fax No: +913262296624, +913262296563).
\end{abstract}

Abstract: Occurrence of arsenic in the groundwater has become a cause for concern in many countries. The presence of As(III) species in the groundwater had been one of the biggest challenges for the water workers especially in the south east Asian countries. Nano based adsorption techniques are gaining attention among researchers for the removal of arsenic ions. However, synthesis of nano-adsorbents is a costly affair. Therefore, the present study utilized the cheap and readily available laterite soil as a base material for nano coating. Nano sized manganese (nMn) particles were synthesized by chemical reduction method and later coated on calcined laterite (Lt-nMn). Coating with $0.1 \mathrm{M}$ nMn provided the best As(III) removal efficiency. The prepared material was characterised for its morphological and surface properties. Phase analysis using XRD (X-ray diffraction) showed the presence of zero valent manganese species, which assisted in adsorption. Adsorption studies were conceded by selecting the different affecting parameters such as contact time, concentration, dose, temperature, and $\mathrm{pH}$. Very high removal in less time regime led to the investigation of the oxidation mechanism. Phosphate and sulphate anions insignificantly reduced the removal efficiency. Langmuir model for the sorption isotherms and pseudo-second order kinetic model for the sorption kinetics symbolized the experimental data. Sustainability studies in terms of eco-scaling and cost analysis were performed for the applied method of production of the nano materials. Adsorber design analysis was also conducted to estimate the required amount of Lt-nMn particles for achieving the desired equilibrium As(III) concentration. 
37 Keywords: Adsorption; As(III) removal; Batch adsorber design; nano Manganese particle; and Laterite.

\section{Introduction}

40

41 Arsenic is a potentially toxic element even at low concentrations, generally found in groundwater due to weathering

42 of arsenic-bearing minerals, overuse of arsenic-contained pesticides, and industrial discharge (Smedley and 43 Kinniburgh, 2002). People of a wide geographic region including several countries such as Bangladesh, India, 44 Vietnam, China, Nepal, Pakistan, Chile, Cambodia, Hungary, Canada, Germany, United States of America, Argentina, 45 and Mexico are suffering from this groundwater-poison (Nguyen et al., 2020 ; Mondal et al., 2008). Arsenite [As(III)] 46 and arsenate $[\mathrm{As}(\mathrm{V})]$ are the two major inorganic arsenic species generally found in natural groundwater of arsenic 47 contaminated areas. The occurrence of these species depends on groundwater chemistry such as redox potential, $\mathrm{pH}$, 48 and concentration of other dissolved ions (Thanh et al., 2016). As(III) predominates in the groundwater where reducing 49 and anaerobic conditions occur due to the presence of organic matter and high sulphate content. Whereas, the As(V) 50 is expected to be present under alkaline environment in arid to semi-arid climates (Mukherjee et al., 2018). As(III) is 51 more toxic and for being a neutral species, it is more difficult to remove by any treatment technology (Zhang et al., 52 2017). Intake of arsenic contaminated water can lead to cancers in different vital organs. Considering the potential 53 carcinogenic effect, the permissible limit of arsenic in drinking water has been recently reduced to $10 \mathrm{ppb}$ from 50 $54 \mathrm{ppb}$ in India (BIS, 2012). A treatment technology that can simultaneously remove both As(III) and As(V) with high 55 removal efficiency is essentially desirable.

56

57 Several arsenic removal technologies such as ion exchange, membrane filtration, adsorption, coagulation and 58 precipitation have been tried by researchers worldwide. Out of these technologies, adsorption is drawing more 59 attention from the researchers owing to the involvement of low cost, high removal efficiency, safer to handle, low 60 sludge production, simplicity in design, and easier applicability in the rural area (Lata et al., 2019).

61

62 In particular, iron-based adsorbents have been the most favoured candidate for arsenic removal. It was also witnessed 63 that the $\mathrm{As}(\mathrm{V})$ is easy to remove from groundwater. Hence, it is most often advised to oxidise $\mathrm{As}(\mathrm{III})$ ions into $\mathrm{As}(\mathrm{V})$

64 before their adsorption. Application of nanomaterials as an adsorbent for arsenic removal is also very trendy and 
popular among water scientists. Nevertheless, the synthesis of these nano materials has always been a matter of concern in terms of eco friendliness and cost. Additionally, the separation after treatment and low permeability in

67 flow-through conditions are some of the inherent problems of nano adsorbent application. This can be avoided if nano adsorbents are incorporated into suitable and cost-effective host matrix such as soil, and sand. Application of manganese and iron in combination can solve the problem associated with the As(III) removal.

71 Manganese and iron oxide minerals are commonly found in soil and reported to have the high oxidizing potential of $72 \mathrm{As}(\mathrm{III})$ and adsorption of arsenic (Wang et al., 2015). The use of Fe-Mn binary oxides for adsorption of arsenic is 73 gaining interest among the researchers as oxides of iron are well reported to have strong affinity and selectivity for 74 arsenic (Wen et al., 2017; Ge et al., 2016). Particularly the nano sized manganese can facilitate the smoother oxidation 75 and adsorption of As(III) ions. Integration of this nano-manganese with the iron particles is seemingly a viable option 76 for arsenic treatment. However, the iron particles need not be present in the nano form. This may increase the cost of 77 synthesis and application. Hence, the readily available and cost-effective source of iron must be selected. Among

78 various iron minerals, laterite is abundantly available in nature and has been extensively studied as a potential 79 adsorbent for arsenic removal (Partey et al., 2008). A combination of nano form of manganese and laterite soil as a 80 host matrix can solve the problems of As(III) removal as well as the nano material application. Thus, for the present

81 study, a noble adsorbent was prepared by coating laterite (Lt) with nano manganese particles (nMn) (Lt-nMn 82 adsorbent) to remove higher amount of As(III) ions from groundwater. The removal behavior of As(III) by Lt-nMn 83 adsorbent was assessed and optimized by observing the effect of each parameter affecting the adsorption process. 84 Severity of the arsenic contamination has sufficiently been discussed in terms of affected population and available 85 resources with them. Thus, it was imperative to assess the developed adsorbent with regard to economic and 86 environmental aspects. Therefore, to evaluate the sustainability, Eco scale and cost factor were also used.

87

\section{Experimental Details}

89

\subsection{Chemicals and Reagents}

91 All chemicals used in this study were of laboratory grade without further purification. All the glassware was 92 thoroughly cleaned with lab wash (neutral) and washed thoroughly with tap water followed by rinsing with deionized 
water and sterilized in hot air oven before use. Type II deionized water (resistivity $=15.0 \mathrm{M} \Omega \cdot \mathrm{cm}$ ) was used throughout the study. The laterite soil was collected from Kharagpur, India.

\subsection{Procedure of synthesizing nano manganese ( $n M n)$ and nMn coated Laterite (Lt-nMn)}

Synthesis of nano Mn was conceded by chemical reduction method using $\mathrm{NaBH}_{4}$ (SRL Chemicals, India) as a reducing agent (Dada et al., 2017). In a typical synthesis, $0.05 \mathrm{M} \mathrm{MnCl}_{2} .4 \mathrm{H}_{2} \mathrm{O}$ (Loba chemie, India) was prepared and transferred into three-necked round bottom flask. 0.2 $\mathrm{M} \mathrm{NaBH}_{4}$ was added dropwise under $\mathrm{N}_{2}$ flow to the former solution. With the addition of $\mathrm{NaBH}_{4}$ the colour of solution changed to brownish colour. Also, the hydrogen gas was evolving. The scheme of synthesis can be observed as per the following reaction (Eq.1).

$$
\mathrm{Mn}^{2+}+2 \mathrm{BH}^{-}+6 \mathrm{H}_{2} \mathrm{O} \rightarrow \mathrm{Mn}^{0}+2 \mathrm{~B}(\mathrm{OH})_{3}+7 \mathrm{H}_{2} \uparrow
$$

The reaction mixture was further stirred for $3 \mathrm{~h}$ and allowed to age overnight. Later, it was filtered using cellulose nitrate membrane filter $(0.22 \mu)$ and washed with ethanol and deionised water. The obtained solid product was oven dried at $60{ }^{\circ} \mathrm{C}$ for $5 \mathrm{~h}$ and kept in a desiccator for further application.

For synthesising the nMn coated laterite, first of all, collected laterite soil was washed with tap water and sun dried. Dried soil particles were crushed and sieved to obtain the particle size of 0.075 to $0.125 \mathrm{~mm}$. Further, it was washed with a copious amount of deionized water and again sun dried. Finally, the soil particles were calcined at $750{ }^{\circ} \mathrm{C}$ for

$1131 \mathrm{~h}$ to remove primarily the organic impurities. In order to coat the $\mathrm{nMn}$, calcined laterite particles were mixed with 114 the manganese chloride solution and the synthesis process remained same as stated above for the nanoparticles. The amount of nMn coating was optimized as a function of As(III) removal.

\subsection{Characterisation studies}

118 The synthesized adsorbent particles were checked for their size, shape, surface area and purity by various techniques 

Infrared Spectroscopy (FTIR) and Zero point charge analysis (pHzpc).

\subsection{Sorption studies}

Sorption studies for the removal of $\mathrm{As}(\mathrm{III})$ ions were performed in batch mode. Sodium (meta) arsenite $\left(\mathrm{NaAsO}_{2}\right)$ (Loba chemie, India) was used to make an As(III) stock solution with a concentration of $1000 \mathrm{mg} / \mathrm{L}$. Working solution of desired concentration was prepared from the stock solution by the dilution method. Adsorption experiments were conducted as a function of affecting parameters such as contact time, initial concentration, dosage, $\mathrm{pH}$, and temperature. The effect of the individual parameter was estimated by one variable at a time approach (OVAT). The variation in the range of parameters was observed as $10-2160 \mathrm{~min}$ for contact time; $100-1000 \mathrm{ppb}$ for initial concentration; 0.25 - $4 \mathrm{~g} / \mathrm{L}$ for dosage; $2-12$ for $\mathrm{pH}$; and 298 - 318K for the temperature. With the optimized value of parameters, isotherm and kinetic experiments were done to know the adsorption behavior and removal mechanism. In addition to it, oxidation study was also performed as a function of time. Removal efficiency in the presence of commonly found anions such as chloride, sulphate, carbonate, and phosphate were also tested for assessing its real performance in the field environment. The longevity of the adsorbent is always desirable for its field application and cost-effectiveness. Thus, a regeneration study was also performed for the prepared adsorbent. $50 \mathrm{~mL}$ solution containing adsorbent and adsorbate was shaken using orbital shaker (Rivotek, India) for a definite time interval. Thereafter, the solution was filtered using syringe filter $(0.22 \mu)$. The remaining As(III) concentration in the solution was measured using hydride generation technique of atomic absorption (HGAA) spectrophotometer (GBC AvantaPM, Australia). During the batch study, adsorption capacity of Lt-nMn particles, and the removal efficiency were 141 calculated using the following equations (Eq. 2 and 3):

$$
Q_{e}=\frac{\left(C_{0}-C_{e}\right) V}{m}
$$

144 Where, $C_{o}$ and $C_{e}$ are the initial and equilibrium concentrations of $A s\left(\right.$ III) in ppb. $Q_{e}$ is the adsorption capacity at 145 equilibrium in $\mu \mathrm{g} / \mathrm{g}, \mathrm{V}$ is the volume of the sample in litres and $\mathrm{m}$ is the weight $(\mathrm{g})$ of $\mathrm{Lt}-\mathrm{nMn}$. 


\section{Results and Discussion}

\subsection{Structural analysis of the adsorbent}

The results obtained from histogram analysis (Fig. 1) showed that the size majority of the particles is in the range of $14.91-118 \mathrm{~nm}$. The moderate degree of stability of the manganese nano particles (n-Mn) in the aqueous solution was indicated by zeta potential value of $-15.4 \mathrm{mV}$ as shown in Fig. 2 (Madhavi et al., 2013). The morphological examination of the adsorbent using FESEM and EDX has been shown in Fig. 3 and Fig.4. The majority of the particles were spherical with aggregation. The size varied from $11-96 \mathrm{~nm}$. Results of EDX analysis confirmed the purity of the adsorbent. Lt-nMn particles showed the presence of Fe, Al, and Si. 3-D topographic image obtained using AFM analysis showed hill-valley-like architecture due to agglomeration of the particles. The results were found consistent with the DLS and FESEM analysis (Fig. 5). The XRD pattern of the adsorbent presented in Fig. 6, shows the strong peaks at $2 \theta$ values of $40.09^{\circ}, 41.48^{\circ}, 42.87^{\circ}, 50.01^{\circ}, 74.12^{\circ}$, and $81.57^{\circ}$ correspond to predominant diffraction peaks of zero valent Mn (ZVM) (Panda et al., 2019). Peaks at $2 \theta$ values of $24.5^{\circ}$ and $38.7^{\circ}$ showed the presence of goethite in the treated laterite sample. Besides, the peaks obtained at $2 \theta$ values of $28.91^{\circ}, 32.42^{\circ}, 38.38^{\circ}, 58.54^{\circ}$; and $47.9^{\circ}$, 53.7 ${ }^{\circ}$ correspond to $\mathrm{Mn}_{3} \mathrm{O}_{4}$ and weakly crystalline ferrihydrite respectively. The FTIR spectra of the adsorbent, before and after adsorption of $\mathrm{As}(\mathrm{III})$ are presented in Fig 7. Intense bands observed in the range of $400-650 \mathrm{~cm}^{-1}$ are ascribed to the Mn-O bending vibrations. The peaks obtained at 628 and $513 \mathrm{~cm}^{-1}$ correspond to the asymmetric bending vibrations of $\mathrm{Mn}-\mathrm{O}$ in $\mathrm{Mn}_{3} \mathrm{O}_{4}$ and $\mathrm{MnO}_{2}$. The peak obtained in the range between $3300-3500 \mathrm{~cm}^{-1}$ and a weak band observed at $1625 \mathrm{~cm}^{-1}$ belong to the stretching and bending vibrations of $\mathrm{O}-\mathrm{H}$ bonding related to the 167 physically-sorbed water on the surface of the adsorbent. The characteristic peaks observed at $1408 \mathrm{~cm}^{-1}$ and $1002 \mathrm{~cm}^{-}$

$168{ }^{1}$, were assigned to the bending vibrations of hydroxyl groups on the metal oxides. After As(III) adsorption on the adsorbent, the peaks at $\sim 1408 \mathrm{~cm}^{-1}, 1625 \mathrm{~cm}^{1,}$ and $1002 \mathrm{~cm}^{-1}$ were observed to be slightly shifted upwards with

170 decreased peak intensities. This primarily suggested that the surface hydroxyl group might be involved during the 171 adsorption process. The peak observed at $820 \mathrm{~cm}^{-1}$, which belongs to the stretching vibration of As(V)-O suggests the 172 oxidation of $\mathrm{As}(\mathrm{III})$ to $\mathrm{As}(\mathrm{V})$ and presence of $\mathrm{As}(\mathrm{V})$ as surface complex, not as a precipitated solid phase (Zhang et 173 al., 2007). The $\mathrm{nMn}$ particles had a surface area of $180 \mathrm{~m}^{2} / \mathrm{g}$. While for Lt-nMn adsorbent, it was found as $79.6 \mathrm{~m}^{2} / \mathrm{g}$. 
174 Several recently reported studies on arsenic removal using $\mathrm{nMn}$ and laterite based adsorbents had been reported to 175 contain lesser surface area values (Panda et al., 2019; Glocheux et al., 2013; Bui et al., 2017). The zero point charge 176 (pHzpc) of the Lt-nMn based adsorbent was found to be 6.82 (Fig. 8), which confirms that the adsorbent was positively 177 charged below pH 6.82.

\subsection{Effect of nMn loading on As(III) removal}

Quantity of nMn loading on laterite may affect the performance of the adsorbent in terms of As(III) removal efficiency.

Therefore, the effect of nMn loading on laterite in different quantity was observed by varying the Mn concentration in the solution during loading operation from $0.01 \mathrm{M}$ to $0.5 \mathrm{M}$. Control experiments with pure $\mathrm{Mn}$ and laterite adsorbent was also performed for comparison. Without nMn loading, the removal of As(III) was found as $96 \%$. However, with the increase in the nMn loading up to $0.1 \mathrm{M}$, the removal efficiency kept on increasing (Fig. 9). With $\mathrm{nMn}$ concentration of more than $0.1 \mathrm{M}$, a constant trend in removal efficiency was observed. Thus, $0.1 \mathrm{M} \mathrm{nMn}$ was selected as the optimum concentration for loading during the preparation of Lt-nMn adsorbent. The calcination of laterite made it porous that helped in achieving the higher removal.

\subsection{Parameters affecting adsorption}

192 Contact time, initial arsenic concentration, adsorbent dose, $\mathrm{pH}$, and temperature are the major parameters that may 193 affect arsenic removal in any adsorption process.

\subsubsection{Contact time}

197 Contact time regulates the economic operation of any pilot-scale application. The effect of the contact time was

198 evaluated with an initial As(III) concentration of $100 \mathrm{ppb}$, adsorbent dose of $1 \mathrm{~g} / \mathrm{L}$, and stirring speed of $150 \mathrm{rpm}$ at neutral condition and at a temperature of $298 \mathrm{~K}$. The contact time was varied in the range of $10-2160 \mathrm{~min}$. From the results of the study (Fig 10), it can be seen that with increment in the reaction time from $10 \mathrm{~min}$ to $120 \mathrm{~min}$, the arsenic 
the contact time of 120 min due to saturation of the adsorption sites. Hence, the optimum contact time for further experiments was taken as $120 \mathrm{~min}$. Lower $\mathrm{C}_{\mathrm{e}}$ value in the initial phase of the experiment indicated the involvement of 204 oxidation process, which was further analysed by lowering the contact time.

\subsubsection{Effect of initial As(III) concentration}

The effect of the initial As(III) concentration on removal efficiency was examined by varying concentration in the range of $100-1000 \mathrm{ppb}$ at $\mathrm{pH}$ 7. Contact time and stirring speed were $120 \mathrm{~min}$ and $150 \mathrm{rpm}$ respectively. The equilibrium arsenic concentration kept on increasing as the initial As(III) concentration increased as shown in Fig. 11.

211 This observation suggested that with increase in the initial concentration, the number of surface sites got exhausted

212 leading to incomplete removal of As(III) with a fixed dose of $1 \mathrm{~g} / \mathrm{L}$. The uptake capacity with the rise of initial arsenic 213 concentration, which was due to the driving force by high initial arsenic concentration to overpower all the mass 214 transfer barriers between the solid and aqueous phases of the contaminant (Ghosh et al., 2019). The percentage 215 removal reached a constant value at the initial concentration of $800 \mathrm{ppb}$. No significant change in the removal 216 efficiency was seen with further increment in the initial As(III) concentration. Optimum initial arsenic concentration 217 was, therefore, selected as $800 \mathrm{ppb}$. It is important to note that with a low initial As(III) concentration of $100 \mathrm{ppb}$, the 218 performance of Lt-nMn adsorbent was very satisfactory, as less than $10 \mathrm{ppb}$ As(total) concentration was achieved 219 after treatment. In natural groundwater, the As(III) concentration is generally less than $800 \mathrm{ppb}$. Thus, the satisfactory 220 performance of the adsorbent even with very less initial arsenic concentration demonstrated its compatibility to 221 decrease the arsenic concentration below WHO permissible limit of $10 \mathrm{ppb}$ for arsenic in drinking water.

\subsubsection{Adsorbent Dose}

224

225 The economy of any treatment system depends on the use of optimized quantity of adsorbent dose. In order to observe 226 the response of the adsorbent dose, experiments were carried out by varying the dose from $0.2-4 \mathrm{~g} / \mathrm{L}$ at $\mathrm{pH}$ 7. Samples 227 with initial As(III) concentration of $800 \mathrm{ppb}$ were agitated for $120 \mathrm{~min}$ at $150 \mathrm{rpm}$. The results shown in Fig. 12 revealed that removal efficiency rapidly increased from $81.8 \%$ to $97.7 \%$ when the dose was escalated from $0.25 \mathrm{~g} / \mathrm{L}$ 
to $1.5 \mathrm{~g} / \mathrm{L}$. On further increment of the adsorbent dose, no substantial change was observed. Therefore, for further studies an adsorbent dose of $1.5 \mathrm{~g} / \mathrm{L}$ was selected as optimum.

\subsubsection{Effect of $p H$}

$\mathrm{pH}$ controls the speciation of arsenic [arsenite As(III) and arsenate $\mathrm{As}(\mathrm{V})]$ as well as the charge developed at the surface of the adsorbent, thus regulates the feasibility of the adsorption process (Jamal Uddin and Jeong, 2020). The response of varying $\mathrm{pH}$ on the removal of $\mathrm{As}(\mathrm{III})$ by Lt-nMn adsorbent was examined over a wide $\mathrm{pH}$ range of 2 - 12. Initial As(III) concentration was $800 \mathrm{ppb}$ with an adsorbent dose of $1.5 \mathrm{~g} / \mathrm{L}$. Weak dependence of As(III) removal on $\mathrm{pH}$ was observed in the $\mathrm{pH}$ range $2-8$ as shown in Fig.13. Around 95\% arsenic removal was obtained in this $\mathrm{pH}$ range. However, with the $\mathrm{pH}$ value more than 8 , the percentage of As(III) removal significantly reduced. As(III) was getting oxidized to $\mathrm{As}(\mathrm{V})$ during adsorption reaction as discussed earlier. $\mathrm{As}(\mathrm{V})$ occurs as an anionic species in the $\mathrm{pH}$ range of $\sim 2-14$ (Ghimire et al., 2003). As the pHzpc value of the adsorbent is 6.82, the adsorbent surface will be positively charged and will adsorb more anions below $\mathrm{pH}$ 6.82. At $\mathrm{pH}$ value > pHzpc, the electrostatic repulsion between the negatively charged adsorbent surface and $\mathrm{As}(\mathrm{V})$ led to significantly less removal of arsenic. At higher $\mathrm{pH}$, the excess concentration of $\mathrm{OH}$ - ion in the solution might impart a competitive effect with arsenic for adsorption on the surface sites of Lt-nMn (Pal et al., 2015). Thus, it can be said that the optimum pH range for As(III) removal is $2-8$. This wide range of working $\mathrm{pH}$ falls in the natural groundwater $\mathrm{pH}(\sim 6.8)$ and indicated to be beneficial in

\subsubsection{Temperature}

The applicability of a developed treatment system is largely influenced by its robustness to work in different temperature conditions. The effect of temperature on As(III) removal was therefore, investigated with a wide variation in temperature and As(III) concentration ranging from $298 \mathrm{~K}$ to $318 \mathrm{~K}$ and 100 - $800 \mathrm{ppb}$ respectively. Contact time of

254120 min, stirring speed of $150 \mathrm{ppm}$ and neutral $\mathrm{pH}$ condition were maintained throughout the experiments. Adsorbent dose of $1.5 \mathrm{~g} / \mathrm{L}$ was added. It is evident from Fig. 14 that as the temperature was raised, the removal efficiency of 
257 at $298 \mathrm{~K}$. Furthermore, the results of this study suggested that low (natural) temperature condition is favorable for

258 carrying out the adsorption process, leading to no requirement of any extra energy input, which in turn reduces the

259 cost of treatment. It is important to note that $97.4 \%$ removal was still achieved with the highest escalated temperature

260 of $318 \mathrm{~K}$. Thus, it can be said that the adsorbent proves to work efficiently in a wide range of temperatures.

261

262

\subsection{Adsorption isotherm}

The adsorbate molecules remain distributed between the solid and liquid phase at equilibrium condition [22]. Adsorption isotherm is a mathematical tool that helps to understand the adsorption equilibrium. In the present study, Langmuir and Freundlich, the two extensively used isotherm models, have been applied for better understanding the interaction between the adsorbent and adsorbate. Experiments were performed at three different temperatures of 298 $\mathrm{K}, 308 \mathrm{~K}$, and $318 \mathrm{~K}$ with the initial As(III) concentration varying from $100 \mathrm{ppb}$ to $800 \mathrm{ppb}$. A fixed adsorbent dose of $1.5 \mathrm{~g} / \mathrm{L}$ was added.

\subsubsection{Langmuir isotherm}

272

273 Langmuir isotherm is used for single solute adsorption system. Adsorption is assumed to be monolayer on to the 274 adsorption sites posing equal adsorption energy (Prabhakar and Samadder, 2020). The non-linear expression of this 275 adsorption model is shown in Eq. (4) (Lata et al., 2019).

$$
Q_{e}=\frac{Q_{m} K_{L} C_{e}}{1+K_{L} C_{e}} \quad \text { Eq. (4) }
$$

279 Where $\mathrm{Q}_{\mathrm{e}}(\mu \mathrm{g} / \mathrm{g})$ represents adsorption capacity at equilibrium concentration $\mathrm{C}_{\mathrm{e}}(\mathrm{ppb})$. $\mathrm{Q}_{\mathrm{m}}$ is the maximum monolayer 280 adsorption capacity and $\mathrm{K}_{\mathrm{L}}(\mathrm{L} / \mu \mathrm{g})$ symbolizes the Langmuir constant related to the energy of sorption. The values of $281 \mathrm{Q}_{\mathrm{m}}$ and $\mathrm{K}_{\mathrm{L}}$ were calculated from the slope and intercept of plot of $\mathrm{C}_{\mathrm{e}}$ and $\mathrm{Q}_{\mathrm{e}}$ respectively (Fig. 15). Table 1 presents 282 the calculated values of isotherm parameters. The value of $\mathrm{K}_{\mathrm{L}}$ was calculated to be $0.050 \mathrm{~L} / \mu \mathrm{g}, 0.040 \mathrm{~L} / \mu \mathrm{g}$, and 0.033 $283 \mathrm{~L} / \mu \mathrm{g}$ at temperatures $298 \mathrm{~K}, 308 \mathrm{~K}$, and $318 \mathrm{~K}$ respectively. Higher $\mathrm{K}_{\mathrm{L}}$ values at lower temperatures indicate that the 

$\mu \mathrm{g} / \mathrm{g}, 1240.06 \mu \mathrm{g} / \mathrm{g}$, and $1184.67 \mu \mathrm{g} / \mathrm{g}$ at $298 \mathrm{~K}, 308 \mathrm{~K}$, and $318 \mathrm{~K}$ respectively.

\subsubsection{Freundlich isotherm}

Freundlich isotherm model is generally applied for heterogeneous adsorption characteristics and sorption sites having non-uniform energies of sorption. Eq. (5) demonstrates the non-linear form of this isotherm model (Rauf et al., 2019).

$$
Q_{e}=K_{f} C_{e}^{1 / n} \quad \text { Eq. (5) }
$$

294 Where, $C_{e}(p p b)$ and $Q_{e}(\mu \mathrm{g} / g)$ have the same meaning as stated earlier. $K_{f}\left\{(\mu \mathrm{g} / \mathrm{g}) /(\mu \mathrm{g} / \mathrm{L})^{1 / n}\right\}$ and $n$ correspond to 295 Freundlich constants, and are related to adsorption capacity and adsorption intensity respectively. Fig. 16 was used to 296 obtain these parameters and is listed in Table 1. The calculated values of $\mathrm{K}_{\mathrm{f}}$ were found to be 80.10, 64.997, and 29757.074 at three different temperatures of $298 \mathrm{~K}, 308 \mathrm{~K}$, and $318 \mathrm{~K}$ respectively. The declined $\mathrm{K}_{\mathrm{f}}$ values with the rise 298 in the temperature indicated that low-temperature condition is preferable for As(III) adsorption using Lt-nMn 299 adsorbent. The calculated values of $1 / \mathrm{n}$ at $298 \mathrm{~K}, 308 \mathrm{~K}$, and $318 \mathrm{~K}$ were $0.763,0.735$, and 0.724 respectively. Values 300 of $1 / \mathrm{n}$ lesser than 1 showed that the adsorption process is favourable (Rauf et al., 2019).

301

Furthermore, the fitness of models was evaluated not only as a function of $\mathrm{R}^{2}$ but also considered error function chisquare $\left(\chi^{2}\right)$ which is represented by Eq. (6).

$$
\chi^{2}=\sum_{i=1}^{n} \frac{\left(q_{\text {ecalc }}-q_{\text {eexp }}\right)^{2}}{q_{\text {eexp }}}
$$

307 Although the $\mathrm{R}^{2}$ values for both the models were found very similar. However, the $\chi^{2}$ values were able to distinguish 308 the model applicability. Langmuir isotherm model yielded lower $\chi^{2}$ values than the Freundlich isotherm. This suggested the applicability of the Langmuir equation for the present study. Langmuir maximum adsorption capacity 
of Lt-nMn adsorbent outperformed several other adsorbents such as Fe (III) - Sn (IV) mixed binary oxide - coated sand, manganese and iron oxide pillared clays, $\alpha-\mathrm{MnO}_{2}$ nanowires, alpha nano alumina, iron impregnated banana pith

312 biochar, silica based catalytic media and laterite soil which were applied for As(III) removal (Table 2).

\subsection{Kinetic studies}

The time required by the adsorbent to uptake the contaminant to reach equilibrium is explained by adsorption kinetics. Kinetic studies also facilitate useful information about the mechanism of adsorption. Experiments were performed with initial As(III) concentration of $800 \mathrm{ppb}$, adsorbent dose of $1.5 \mathrm{~g} / \mathrm{L}$, and a wide range of temperature (298 K - 318 K). Samples were shaken for different time intervals (5 min - $120 \mathrm{~min}$ ) at $150 \mathrm{rpm}$ and at neutral $\mathrm{pH}$.

\subsubsection{Pseudo-first order kinetic model}

$$
Q_{t}=Q_{e}\left(1-\mathrm{e}^{-k_{1} \mathrm{t}}\right)
$$

327 Where, $\mathrm{Q}_{\mathrm{e}}$ and $\mathrm{Q}_{\mathrm{t}}$ (both in $\mu \mathrm{g} / \mathrm{g}$ ) depict the amount of arsenic adsorbed at equilibrium and any time $\mathrm{t}$ respectively.

328 Theoretical adsorption capacity and pseudo-first-order rate constant $\left(\mathrm{k}_{1}\right)$ were obtained from the slope and intercept 329 respectively of the plot of $\mathrm{Q}_{t} \mathrm{vs.}$ time (t) as shown in Fig. 17. From the rate constants shown in Table 3, it can be seen that $\mathrm{k}_{1}$ varied from $0.809 \mathrm{~min}^{-1}$ to $0.684 \mathrm{~min}^{-1}$ with the increase in the temperature from 298 to $318 \mathrm{~K}$. The lower $\mathrm{R}^{2}$ value obtained in this model suggested that pseudo-first-order model could not be applied as kinetic model for the 332 present study.

\subsubsection{Pseudo-second-order kinetic model} Eq. (8). 


$$
Q_{t}=\frac{k_{2} Q_{e}{ }^{2} t}{1+k_{2} Q_{e} t}
$$

341 Where, $\mathrm{Q}_{\mathrm{e}}$ and $\mathrm{Q}_{\mathrm{t}}$ bear a similar meaning as stated before. $\mathrm{K}_{2}$ is the pseudo-second order rate constant and obtained

342 from the plot of $Q_{t}$ vs. $t$ [Fig. 18]. The obtained $R^{2}$ value was found satisfactory as can be seen from Table 2. Hence,

343 pseudo-second order kinetic model did explain the adsorption kinetic for the present study. In order to identify the rate

344 limiting step in the adsorption process, intra-particular and liquid film diffusion model was employed.

\subsubsection{Intra-particular diffusion}

The assumptions of this model are, the adsorbate travels to the pore of the adsorbent after being primarily surface adsorbed and adsorption capacity is directly proportional to $t^{0.5}$. The mathematical expression of this model is given by Eq. (9) (Morris and Weber, 1963).

$$
Q_{t}=k_{i d} t^{0.5}+C
$$

354 Where, $\mathrm{k}_{\mathrm{id}}$ and $\mathrm{C}$ denote intra-particular diffusion rate constant and intercept of the graph respectively as shown in 355 Fig. 19. It showed that the straight line did not cross through the origin and a multi-segmented plot was obtained, 356 which suggests that intra-particular diffusion is not a rate-limiting step for the current study. The high value obtained 357 for the intercept at three different temperatures is a preliminary indication of the role of the boundary layer for the 358 adsorption process (Khan et al., 2013).

\subsubsection{Liquid film diffusion}

362 As the intra particular diffusion model did not clearly explain the rate limiting step for the current study, liquid film 363 diffusion model was employed to the experimental data of the kinetic study. The model is expressed by Eq. (10) 364 (Weber and Chakravorti, 1974). 


$$
\ln (1-f)=-k_{f d} t
$$

368 Where, the fractional achievement of equilibrium is denoted by $f=\mathrm{Q}_{\mathrm{t}} / \mathrm{Q}_{\mathrm{e}}$ and $\mathrm{k}_{\mathrm{fd}}$ is the rate constant of liquid film 369 diffusion. A straight line was produced at all four temperatures as can be seen from the plot of $\ln (1-\mathrm{f})$ vs. $\mathrm{t}$ [Fig. 20]. 370 At $298 \mathrm{~K}, 318 \mathrm{~K}$, and $308 \mathrm{~K}$ the $\mathrm{k}_{\mathrm{fd}}$ values were $-0.011,-0.029$, and -0.030 respectively. The higher $\mathrm{R}^{2}(0.95$ to 0.89$)$ 371 and lower intercepts (-3.4 to -3.23) obtained in this model than that of intra-particle diffusion, suggested that the 372 formation of a film of oxides of $\mathrm{Mn}$ and $\mathrm{Fe}$ on the adsorbent surface might control the adsorption kinetics for the 373 present study (Table 4).

\subsection{Thermodynamic studies}

Temperature related experimental data were further analyzed to calculate different thermodynamic parameters such as standard free energy $\left(\Delta \mathrm{G}^{\circ}\right)$, standard entropy $\left(\Delta \mathrm{H}^{\circ}\right)$ and standard enthalpy $\left(\Delta \mathrm{S}^{\circ}\right)$ for a better understanding of the adsorption mechanism. Different parameters of thermodynamic study have been shown in Eq. (11) - (13) (Nassar et al., 2017).

$$
\Delta G^{\circ}=-R T \ln K_{c} \quad \text { Eq. (11) }
$$

Where, $R$ denotes the universal gas constant $(\mathrm{kJ} / \mathrm{mol} . \mathrm{K}), \mathrm{T}(\mathrm{K})$ is the temperature, $\mathrm{K}_{\mathrm{c}}$ represents solute distribution coefficient and was obtained from the ratio of $\mathrm{Q}_{\mathrm{e}} / \mathrm{C}_{\mathrm{e}}$. The slope and intercept of the plot between $\ln \mathrm{K}_{\mathrm{c}} \mathrm{vs.}$ 1/T (Fig. 21) yielded the value of $\Delta \mathrm{H}^{\circ}$ and $\Delta \mathrm{S}^{\circ}$ respectively and presented in Table 5. Standard enthalpy $\left(\Delta \mathrm{H}^{\circ}\right)$ and entropy values $\left(\Delta \mathrm{S}^{\circ}\right)$ were calculated as $-21.27 \mathrm{~kJ} / \mathrm{mol}$ and $-0.04 \mathrm{~kJ} / \mathrm{mol} . \mathrm{K}$. The $\Delta \mathrm{H}^{\circ}$ value less than $40 \mathrm{~kJ} / \mathrm{mol}$ suggests that the adsorption process might be physio-sorption. Negative $\Delta \mathrm{S}^{\circ}$ value indicated that the randomness of the solute [As(III)] 
decreased in the solution as the concentration of the adsorbate increased on the surface of the adsorbent. At three different temperatures of $298 \mathrm{~K}, 318 \mathrm{~K}$, and $308 \mathrm{~K}$, the standard free energy $\left(\Delta \mathrm{G}^{\circ}\right)$ was found to be $-9.34 \mathrm{~kJ} / \mathrm{mol}$, $8.94 \mathrm{~kJ} / \mathrm{mol}$, and $-8.54 \mathrm{~kJ} / \mathrm{mol}$ respectively. These negative values of $\Delta \mathrm{G}^{\circ}$ at all three temperatures further exhibited the spontaneous and favorable nature of adsorption. The declined value of $\Delta \mathrm{G}^{\circ}$ with the rise in the temperature further showed that the adsorption process is less likely to occur at higher temperatures. Furthermore, in the present study, $\Delta \mathrm{G}^{\circ}$ was found to be in the range of 0 to $-20 \mathrm{~kJ} / \mathrm{mol}$, which made the process to be physio-sorption (Nassar and Khatab, 2016).

\subsection{Investigation of possible oxidation of $\mathrm{As}(\mathrm{III})$ to $\mathrm{As}(\mathrm{V})$ by Lt-nMn particles}

Oxidation of As(III) was evaluated with an initial As(III) concentration of $100 \mathrm{ppb} .1 .5 \mathrm{~g} / \mathrm{L}$ adsorbent dose was added and samples were shaken for a different time interval ranging from 1 to 10 min. Fig. 22 shows that the concentration of As(III) kept on decreasing as the reaction progressed, whereas, $\mathrm{As}(\mathrm{V})$ concentration was increasing. It was observed that till $10 \mathrm{~min}$ of contact time, $\mathrm{As}(\mathrm{V})$ concentration increased. Interestingly, after $7 \mathrm{~min}$, the total arsenic concentration started decreasing, which was the mark of the initiation of adsorption process. The oxidation of As(III) into $\mathrm{As}(\mathrm{V})$ can, therefore, be due to the formation of oxides of manganese on the adsorbent surface, mainly $\mathrm{MnO}_{2}$. The observed characteristic band for As(V)-O during the FTIR study of the adsorbent sample after adsorption also supported this

\subsection{Effect of coexisting ions}

412 Anions such as phosphate, chloride, sulphate, and carbonate are generally found to be present in groundwater and 413 reported to influence the arsenic removal efficiency due to their competitive effects on the binding sites of the adsorbent (Maiti et al., 2012). The effects of these competing coexisted ions were evaluated at a high concentration

415 (2000 ppb) of these anions separately. An optimum adsorbent dose of $1.5 \mathrm{~g} / \mathrm{L}$ was added to $800 \mathrm{ppb}$ initial As(III) 416 concentration at $\mathrm{pH}$ 7. From the results of this study shown in Fig. 23, it was observed that the chloride and carbonate 417 did not noticeably affect the removal efficiency of the adsorbent. Whereas, in the presence of sulphate and phosphate, 418 As(III) removal efficiency was reduced by $1 \%$ and $1.7 \%$ respectively. The slight reduction in the removal efficiency 
419 in the presence of these co-ions suggested that the oxidation of As(III) into As(V) gave a preferential selectivity to 420 arsenic adsorption over phosphate, indicating higher affinity of the Lt-nMn towards $\mathrm{As}(\mathrm{V})$ species. Furthermore, it 421 can be said that Lt-nMn has a good potential in treating arsenic containing groundwater with a high concentration of 422 coexisting anions.

\subsection{Regeneration of Lt-nMn adsorbent}

Reusability of any adsorbent directly controls the economic feasibility of that particular treatment technology. Thus,

427 a regeneration study of the adsorbent was done to establish its reusing capability and economic feasibility of the 428 treatment system. The adsorbent was regenerated by raising the $\mathrm{pH}$ above the zero point charge of the adsorbent $(\mathrm{pHzpc}=6.8)$ using $0.1 \mathrm{~N} \mathrm{NaOH}$. Above pHzpc, the negatively charged adsorbent surface desorbed As(III) oxyanion. Four adsorption-desorption cycles were carried out with $800 \mathrm{ppb}$ initial As(III) concentration (Fig. 24). Only 3\% reduction in the removal efficiency was observed at the end of $4^{\text {th }}$ cycle, which suggests that the adsorbent has a very high reusable capacity that may reduce the treatment cost to a great extent.

\subsection{Eco-Scale and cost analysis for the synthesis of Lt-nMn adsorbent}

Table 5a shows eco-scale analysis for the synthesis of Lt-nMn adsorbent for arsenic removal. The penalty points for each material were allocated based on the safety, cost, and energy requirement (Medina-Ramirez et al., 2019). In the present study, eco-scale value for the method of synthesis of Lt-nMn particles was found as $86 \%$. Generally, the ecoscale value greater than $75 \%$ is considered as a green process. Additionally, the environment factor (E factor) and

440 water intensity were calculated as 0.008 and 34.37 respectively.

$441 \mathrm{E}$ factor $=[\mathrm{kg}($ raw materials $)-\mathrm{kg}($ desired product $)] / \mathrm{kg}$ (total product including water) Eq.(14)

442 Water intensity $(\mathrm{Wp})=$ (mass of all water used/ mass of product) $\mathrm{kg} / \mathrm{kg}$ product Eq. (15) positive, since, for every one litre of water consumed for synthesis, nearly $220 \mathrm{~L}$ of water can be treated. The 

the synthesis process is feasible from economic point of view.

\subsection{Amount estimation study using adsorber design analysis}

451

452 Apart from sustainability analysis, it is equally important to quantify the required amount of Lt-nMn particles for treating the known volume of arsenic-contaminated water. Design of single stage batch adsorber by mass balance approach, can help in estimation of the amount. The results of isotherm adsorption experiment were applied to the following equations (Eq.16) to find the required dosage (Prabhakar and Samadder, 2020).

$$
\frac{M}{V}=\frac{C_{1}-C_{e}}{Q_{e}}
$$

The $\mathrm{Q}_{\mathrm{e}}$ value in Eq. 16 can be found using the Langmuir isotherm, as this was the governing isotherm model in the present study. Therefore, Eq. 16 can be rewritten as,

$$
\frac{M}{V}=\frac{C_{1}-C_{e}}{\frac{Q_{m} K_{L} C_{e}}{1+K_{L} C_{e}}} \quad \text { Eq. (17) }
$$

463 Where the $C_{1}$ and $C_{e}$ are the initial and desired equilibrium arsenic concentration. If the values of $C_{1}, C_{e}, Q_{e}$, and V are 464 known, then with the help of Eq. (17), mass of Lt-nMn can easily be calculated. For a hypothetical situation, the equilibrium concentration was fixed at $5 \mathrm{ppb}$ for different concentrations ranging from 100 to $500 \mathrm{ppb}$. The range of 466 chosen concentration mimics the natural arsenic concentration found in this region. Fig. 25 shows the calculated 467 amount of Lt-nMn particles for different volumes of water to be treated. For treating the $1000 \mathrm{~L}$ water having $200 \mathrm{ppb}$ 468 concentration, approximately $0.70 \mathrm{~kg}$ of $\mathrm{Lt}-\mathrm{nMn}$ is required. Out of this amount, much of the portion consists of 469 laterite soil particles. Also, if regeneration efficiency is taken into consideration then nearly 4 times higher volume of 470 water can be treated with the same amount of Lt-nMn particles. 
474 This study reported the efficient and cost-effective method for the synthesis of nano Mn coated laterite particles, that 475 were applied for As(III) removal from groundwater. Laterite soil as a base material for nano Mn coating not only 476 provided the synergism for removal but also provided a way to think beyond the sole use of nano materials for removal 477 processes. Coating of $\mathrm{nMn}$ was optimised as a function of As(III) removal efficiency. With pure nMn and laterite 478 particles, less removal was observed. The calcination process removed the organic impurities and thus hardness and 479 porosity were improved. This eventually prevented the disintegration and adhesion in water. Characterisation studies 480 using FESEM analysis revealed that synthesized nMn particles were spherical and of nano size range. EDX analysis 481 further confirmed the purity of nMn. Surface area of Lt-nMn was found lower as compared to nMn which further 482 assured that coating was successfully achieved. Results of adsorption studies reflected that the up to $700 \mathrm{ppb}$ of initial 483 As(III) concentration, remaining concentration in the solution was brought down below the 10 ppb mark. Oxidation 484 of $\mathrm{As}(\mathrm{III})$ species facilitated the smooth removal arsenic ions in the form of $\mathrm{As}(\mathrm{V})$. The removal was insignificantly 485 affected till $\mathrm{pH}$ 7. Adsorption isotherm was clarified satisfactorily by Langmuir isotherm with a maximum monolayer 486 adsorption capacity of $1376.882 \mu \mathrm{g} / \mathrm{g}$ at $298 \mathrm{~K}$. Isotherm and thermodynamic studies indicated an exothermic nature 487 of adsorption. Adsorption was less likely to be affected by the presence of commonly found anions in the groundwater. 488 Furthermore, the sustainability studies confirmed that the Lt-nMn had very less potential to cause any harm to the 489 environment.

\section{Acknowledgements}

Authors express thanks the Science \& Engineering Research Board (A Statutory body of Department of Science and 494 Technology, Govt. of India) (Project No. SB/EMEQ-010/2014) for financially supporting this work. The authors 495 would also like to acknowledge the support provided by the Department of Environmental Science \& Engineering, Indian Institute of Technology (Indian School of Mines), Dhanbad, India.

\section{Ethical approval and consent to participate}




\section{Consent to publish}

501

Not Applicable

502

\section{Authors' contributions}

503

Conceptualization: Roshan Prabhakar; Methodology: Roshan Prabhakar, Somaparna Ghosh, Ali; Formal analysis

and investigation: Somaparna Ghosh, Ali; Writing - original draft preparation: Roshan Prabhakar, Somaparna

Ghosh; Writing - review and editing: Roshan Prabhakar, Sukha Ranjan Samadder; Funding acquisition: Sukha

Ranjan Samadder; Resources: Sukha Ranjan Samadder; Supervision: Sukha Ranjan Samadder, Roshan Prabhakar

\section{Competing interests}

The authors declare that they have no competing interests.

Availability of data and materials

All data generated or analysed during this study are included in this manuscript.

\section{References}

Aremu JO, Lay M, Glasgow G. Kinetic and isotherm studies on adsorption of arsenic using silica based catalytic media. Journal of Water Process Engineering. 2019 Dec 1;32:100939.

BIS, I. (2012). 10500: 2012. Indian standard drinking water-specification (second revision), Bureau of Indian Standards, New Delhi.

Bui TH, Kim C, Hong SP, Yoon J. Effective adsorbent for arsenic removal: core/shell structural nano zero-valent iron/manganese oxide. Environmental Science and Pollution Research. 2017 Nov;24(31):24235-42. use for the removal of As (III) and As (V) from water: application of isotherm, kinetic and thermodynamics. Journal of Molecular Liquids. 2016 Dec 1;224:431-41.

Dada AO, Adekola FA, Odebunmi EO. A novel zerovalent manganese for removal of copper ions: synthesis, characterization and adsorption studies. Applied Water Science. 2017 Jun 1;7(3):1409-27. 
Foroutan, Rauf, Reza Mohammadi, Adeyemi S. Adeleye, Sima Farjadfard, Zahra Esvandi, Hossein Arfaeinia, George A. Sorial, Bahman Ramavandi, and Soleyman Sahebi. "Efficient arsenic (V) removal from contaminated water using natural clay and clay composite adsorbents." Environmental Science and Pollution Research 26, no. 29 (2019): 29748-29762.

Ge X, Liu J, Song X, Wang G, Zhang H, Zhang Y, Zhao H. Hierarchical iron containing $\gamma$-MnO2 hollow microspheres: a facile one-step synthesis and effective removal of As (III) via oxidation and adsorption. Chemical Engineering Journal. 2016 Oct 1;301:139-48.

Ghimire KN, Inoue K, Makino K, Dhakal RP. Adsorptive removal of arsenic and fluoride by using orange juice residue. Electrometallurgy and Environmental Hydrometallurgy. 2003 Aug 1;2:1937-50.

Ghosh S, Prabhakar R, Samadder SR. Performance of $\gamma$-aluminium oxide nanoparticles for arsenic removal from groundwater. Clean Technologies and Environmental Policy. 2019 Jan 15;21(1):121-38.

Glocheux Y, Pasarín MM, Albadarin AB, Allen SJ, Walker GM. Removal of arsenic from groundwater by adsorption onto an acidified laterite by-product. Chemical Engineering Journal. 2013 Jul 15;228:565-74.

Ho YS, McKay G. A comparison of chemisorption kinetic models applied to pollutant removal on various sorbents. Process safety and environmental protection. 1998 Nov 1;76(4):332-40.

Khan TA, Chaudhry SA, Ali I. Thermodynamic and kinetic studies of As (V) removal from water by zirconium oxidecoated marine sand. Environmental Science and Pollution Research. 2013 Aug;20(8):5425-40.

Lata S, Prabhakar R, Adak A, Samadder SR. As (V) removal using biochar produced from an agricultural waste and prediction of removal efficiency using multiple regression analysis. Environmental Science and Pollution Research. 2019 Nov;26(31):32175-88.

Lou Z, Cao Z, Xu J, Zhou X, Zhu J, Liu X, Baig SA, Zhou J, Xu X. Enhanced removal of As (III)/(V) from water by simultaneously supported and stabilized Fe-Mn binary oxide nanohybrids. Chemical Engineering Journal. 2017 Aug 15;322:710-21.

Madhavi V, Prasad TN, Reddy AV, Reddy BR, Madhavi G. Application of phytogenic zerovalent iron nanoparticles in the adsorption of hexavalent chromium. Spectrochimica Acta Part A: Molecular and Biomolecular Spectroscopy. 2013 Dec 1;116:17-25. 
Maiti A, Basu JK, De S. Experimental and kinetic modeling of As (V) and As (III) adsorption on treated laterite using synthetic and contaminated groundwater: Effects of phosphate, silicate and carbonate ions. Chemical Engineering Journal. 2012 May 15;191:1-2.

Medina-Ramirez A, Gamero-Melo P, Ruiz-Camacho B, Minchaca-Mojica JI, Romero-Toledo R, Gamero-Vega KY. Adsorption of aqueous As (III) in presence of coexisting ions by a green Fe-modified W zeolite. Water. 2019 Feb;11(2):281.

Mishra T, Mahato DK. A comparative study on enhanced arsenic (V) and arsenic (III) removal by iron oxide and manganese oxide pillared clays from ground water. Journal of Environmental Chemical Engineering. 2016 Mar 1;4(1):1224-30.

Mondal P, Majumder CB, Mohanty B. Effects of adsorbent dose, its particle size and initial arsenic concentration on the removal of arsenic, iron and manganese from simulated ground water by $\mathrm{Fe} 3+$ impregnated activated carbon. Journal of Hazardous Materials. 2008 Feb 11;150(3):695-702.

Morris WJ, Weber JC. Kinetics of adsorption on carbon from solution. J. Saint. Eng. Div. ASCE. 1963;89:31.

Mukherjee S, Kumar AA, Sudhakar C, Kumar R, Ahuja T, Mondal B, Srikrishnarka P, Philip L, Pradeep T. Sustainable and affordable composites built using microstructures performing better than nanostructures for arsenic removal. ACS Sustainable Chemistry \& Engineering. 2018 Dec 10;7(3):3222-33.

Nassar MY, Khatab M. Cobalt ferrite nanoparticles via a template-free hydrothermal route as an efficient nanoadsorbent for potential textile dye removal. RSC advances. 2016;6(83):79688-705.

Nassar MY, Mohamed TY, Ahmed IS, Samir I. MgO nanostructure via a sol-gel combustion synthesis method using different fuels: an efficient nano-adsorbent for the removal of some anionic textile dyes. Journal of Molecular Liquids. 2017 Jan 1;225:730-40.

Nguyen TH, Tran HN, Vu HA, Trinh MV, Nguyen TV, Loganathan P, Vigneswaran S, Nguyen TM, Vu DL, Nguyen TH. Laterite as a low-cost adsorbent in a sustainable decentralized filtration system to remove arsenic from groundwater in Vietnam. Science of The Total Environment. 2020 Jan 10; 699:134267.

Pal S, Patra AS, Ghorai S, Sarkar AK, Mahato V, Sarkar S, Singh RP. Efficient and rapid adsorption characteristics of templating modified guar gum and silica nanocomposite toward removal of toxic reactive blue and Congo red dyes. Bioresource technology. 2015 Sep 1; 191:291-9. 
Panda AP, Rout P, Jena KK, Alhassan SM, Kumar SA, Jha U, Dey RK, Swain SK. Core-shell structured zero-valent manganese (ZVM): a novel nanoadsorbent for efficient removal of As (iii) and As (v) from drinking water. Journal of Materials Chemistry A. 2019; 7(16):9933-47.

Partey F, Norman D, Ndur S, Nartey R. Arsenic sorption onto laterite iron concretions: temperature effect. Journal of colloid and interface science. 2008 May 15; 321(2):493-500.

Prabhakar R, Samadder SR. Low cost and easy synthesis of aluminium oxide nanoparticles for arsenite removal from groundwater: a complete batch study. Journal of Molecular Liquids. 2018 Jan 1; 250:192-201.

Prabhakar R, Samadder SR. Use of adsorption-influencing parameters for designing the batch adsorber and neural network-based prediction modelling for the aqueous arsenate removal using combustion synthesised nanoalumina. Environmental Science and Pollution Research. 2020 Jul;27(21):26367-84.

Smedley PL, Kinniburgh DG. A review of the source, behaviour and distribution of arsenic in natural waters. Applied geochemistry. 2002 May 1;17(5):517-68.

Thanh DN, Bastl Z, Černá K, Ulbrich P, Lederer J. Amorphous nanosized Al-Ti-Mn trimetal hydrous oxides: synthesis, characterization and enhanced performance in arsenic removal. RSC advances. 2016;6(103):100732-42

Uddin, Md Jamal, and Yeon-Koo Jeong. "Efficiently performing periodic elements with modern adsorption technologies for arsenic removal." Environmental Science and Pollution Research (2020): 1-25.

Wang S, Gao B, Li Y, Mosa A, Zimmerman AR, Ma LQ, Harris WG, Migliaccio KW. Manganese oxide-modified biochars: preparation, characterization, and sorption of arsenate and lead. Bioresource technology. 2015 Apr $1 ; 181: 13-7$.

Weber TW, Chakravorti RK. Pore and solid diffusion models for fixed-bed adsorbers. AIChE Journal. 1974 Mar;20(2):228-38.

Wei Z, Yan J, Lan B, Yu L, Liu Y, Wu Y, Liang K, Pan Z, Hu G, Xian H. Adsorption and Oxidation of Arsenic by Ultra-long $\alpha-\mathrm{MnO} 2$ Nanowires with the $\left(\begin{array}{lll}1 & 1 & 0\end{array}\right)$ Surface. Inorganic and Nano-Metal Chemistry. 2017 Feb 7(just-accepted).

Wen Z, Zhang Y, Wang Y, Li L, Chen R. Redox transformation of arsenic by magnetic thin-film MnO2 nanosheetcoated flowerlike Fe3O4 nanocomposites. Chemical Engineering Journal. 2017 Mar 15;312:39-49. 
605 606

607

608
Zhang G, Xu X, Ji Q, Liu R, Liu H, Qu J, Li J. Porous nanobimetallic Fe-Mn cubes with high valent Mn and highly efficient removal of arsenic (III). ACS applied materials \& interfaces. 2017 May 3;9(17):14868-77.

Zhang GS, Qu JH, Liu HJ, Liu RP, Li GT. Removal mechanism of As (III) by a novel Fe- Mn binary oxide adsorbent: oxidation and sorption. Environmental Science \& Technology. 2007 Jul 1;41(13): 4613-9. 
Table 1: Values of the adsorption isotherm parameters for As(III) using Lt-nMn adsorbent

\begin{tabular}{|c|c|c|c|c|}
\hline \multirow{2}{*}{$\begin{array}{c}\text { S. } \\
\text { No }\end{array}$} & Isotherm models & \multicolumn{2}{|c|}{ Temperature (K) } \\
\cline { 2 - 5 } & & $\mathbf{2 9 8}$ & $\mathbf{3 0 8}$ & $\mathbf{3 1 8}$ \\
\hline \multirow{3}{*}{1.} & Langmuir isotherm & & 1240.061 & 1184.671 \\
\cline { 2 - 5 } & $\mathrm{Q}_{\mathrm{m}}(\mu \mathrm{g} / \mathrm{g})$ & 1376.882 & 0.040 & 0.033 \\
\cline { 2 - 5 } & $\mathrm{K}_{\mathrm{L}}(\mathrm{L} / \mu \mathrm{g})$ & 0.050 & 0.993 & 0.993 \\
\cline { 2 - 5 } & $\mathrm{R}^{2}$ & 0.996 & 6.60 & 8.40 \\
\cline { 2 - 5 } & $\chi^{2}$ & 2.02 & 64.997 & 57.074 \\
\hline \multirow{3}{*}{2.} & Freundlich isotherm & & 0.735 & 0.724 \\
\cline { 2 - 5 } & $\mathrm{K}_{\mathrm{f}}\left\{(\mu \mathrm{g} / \mathrm{g}) /(\mu \mathrm{g} / \mathrm{L})^{1 / \mathrm{n}}\right\}$ & 80.101 & 0.998 & 0.990 \\
\cline { 2 - 5 } & $1 / \mathrm{n}$ & 0.763 & 7.31 & 9.63 \\
\cline { 2 - 5 } & $\mathrm{R}^{2}$ & 0.993 & 3.89 & \\
\cline { 2 - 5 } & $\chi^{2}$ & & & \\
\hline
\end{tabular}


Table 2: Adsorption capacities of various adsorbents used for As (III) removal

\begin{tabular}{|c|c|c|}
\hline Adsorbent & $\begin{array}{c}\text { Maximum adsorption capacity } \\
(\boldsymbol{\mu g} / \mathbf{g})\end{array}$ & Reference \\
\hline $\begin{array}{c}\mathrm{Fe}(\mathrm{III})-\mathrm{Sn}(\mathrm{IV}) \text { mixed binary } \\
\text { oxide }- \text { coated sand }\end{array}$ & 82.64 & Chaudhary et al., (2016) \\
\hline $\begin{array}{c}\text { Manganese oxide pillared clays } \\
\text { Iron oxide pillared clays }\end{array}$ & 25.77 & Mishra and Mahato, (2016) \\
\hline$\alpha$-MnO 2 Nanowires & 17.57 & Mishra and Mahato, (2016) \\
\hline $\begin{array}{c}\text { Alpha nano alumina } \\
\text { Iron impregnated banana pith } \\
\text { biochar }\end{array}$ & 1012 & $\begin{array}{c}\text { Prabhakar and Samadder, } \\
(2018)\end{array}$ \\
\hline $\begin{array}{c}\text { silica based } \\
\text { catalytic media }\end{array}$ & 500 & $\begin{array}{c}\text { Lata et al., (2019) } \\
\text { Laterite soil }\end{array}$ \\
\hline Lt-nMN particles & 315 & Aremu et al.,(2019) \\
\hline & 602 & Present study \\
\hline
\end{tabular}


Table 3: Values of the kinetic parameters for As(III) using Lt-nMn adsorbent

\begin{tabular}{|c|c|c|c|c|}
\hline \multirow{2}{*}{ S. No } & Applied Kinetic Models & \multicolumn{3}{|c|}{ Temperature (K) } \\
\cline { 2 - 5 } & & $\mathbf{2 9 8}$ & $\mathbf{3 0 8}$ & $\mathbf{3 1 8}$ \\
\hline \multirow{3}{*}{1.} & Pseudo-first-order & & & \\
\cline { 2 - 5 } & $\mathrm{Q}_{\mathrm{m}}(\mu \mathrm{g} / \mathrm{g})$ & 517.530 & 515.293 & 513.124 \\
\cline { 2 - 5 } & $\mathrm{k}_{1}$ & 0.809 & 0.862 & 0.684 \\
\cline { 2 - 5 } & $\mathrm{R}^{2}$ & 0.60 & 0.48 & 0.72 \\
\cline { 2 - 5 } & $\chi^{2}$ & 0.060 & 0.064 & 0.066 \\
\hline \multirow{3}{*}{2.} & Pseudo-second-order & 520.564 & 518.118 & 517.867 \\
\cline { 2 - 5 } & $\mathrm{Q}_{\mathrm{m}}(\mu \mathrm{g} / \mathrm{g})$ & 0.140 & 0.124 & 0.236 \\
\cline { 2 - 5 } & $\mathrm{k}_{2}$ & 0.89 & 0.82 & 0.019 \\
\cline { 2 - 5 } & $\mathrm{R}^{2}$ & 0.002 & 0.014 & \\
\hline
\end{tabular}


Table 4: Intra particle and liquid film diffusion parameters for As(III) adsorption using Lt-nMn adsorbent

\begin{tabular}{|c|c|c|c|c|c|c|c|}
\hline S. & Temperature & \multicolumn{3}{|c|}{ Intra particle diffusion } & \multicolumn{3}{c|}{ Liquid film diffusion } \\
\cline { 3 - 8 } No. & $(\mathbf{K})$ & $K_{i d}\left(\mu \mathrm{g}_{\mathrm{g}}^{-1} \cdot \mathrm{min}^{-1}\right)$ & Intercept & $\mathrm{R}^{2}$ & $K_{f d}\left(\mathrm{~g} \cdot \mu^{-1}\right)$ & Intercept & $\mathrm{R}^{2}$ \\
\hline 1. & 298 & 10.11 & 506.37 & 0.93 & -0.011 & -3.40 & 0.95 \\
\hline 2. & 308 & 9.39 & 504.27 & 0.96 & -0.029 & -3.47 & 0.98 \\
\hline 3. & 318 & 9.36 & 496.65 & 0.81 & -0.030 & -3.23 & 0.89 \\
\hline
\end{tabular}


Table 5: Thermodynamic parameters of the adsorption process for As(III) removal using Lt-nMn adsorbent

\begin{tabular}{|c|c|c|c|c|}
\hline S. No. & Temperature (K) & $\Delta \mathbf{G}^{\circ} \mathbf{( k J / m o l )}$ & $\Delta \mathbf{H}^{\circ}(\mathbf{k J} / \mathbf{m o l})$ & $\Delta \mathbf{S}^{\circ}(\mathbf{k J} / \mathbf{m o l} \mathbf{~ K})$ \\
\hline 1. & 298 & -9.34 & & \multirow{2}{*}{-21.27} \\
\cline { 1 - 3 } 2. & 308 & -8.94 & -0.040 \\
\hline 3. & 318 & -8.54 & & \\
\hline
\end{tabular}


Table 6: Sustainability assessment for the synthesis of Lt-nMn adsorbent particles (A) Eco-Scale and (B) Cost

(B) Eco-Scale

\begin{tabular}{|c|c|c|}
\hline Parameter & Amount & $\begin{array}{c}\text { Penalty } \\
\text { Points (PP) }\end{array}$ \\
\hline Laterite & $<10 \mathrm{~g}$ & 0 \\
\hline $\mathrm{MnCl}_{2} \cdot 4 \mathrm{H}_{2} \mathrm{O}$ & $<10 \mathrm{~g}$ & 1 \\
\hline $\mathrm{NaBH}_{4}$ & $<10 \mathrm{~g}$ & 2 \\
\hline Ethanol & $10-100 \mathrm{ml}$ & 4 \\
\hline Waste & $<1 \mathrm{~g}$ & 1 \\
\hline Common setup & ---- & 0 \\
\hline Inert Gas atmosphere & ---- & 1 \\
\hline Room temperature $<24 \mathrm{~h}$ & ------ & 1 \\
\hline Adding solvent & ---- & 3 \\
\hline Heating $>1 \mathrm{~h}$ & --- & 1 \\
\hline AAS & --- & 14 \\
\hline Total Penalty Points (TPP) & & $(100-14)=86$ \\
\hline Eco-Scale & &
\end{tabular}

\section{(B) Cost}

\begin{tabular}{|l|l|l|l|l|}
\hline Reagent & Amount (g) & Amount (ml) & Price (USD) & Price (USD/g) \\
\hline Laterite & 1 & ---- & 0 & 0 \\
\hline $\mathrm{MnCl}_{2} \cdot 4 \mathrm{H}_{2} \mathrm{O}$ & 0.49 & ---- & 0.01 & 0.0204 \\
\hline $\mathrm{NaBH}_{4}$ & 0.60 & --- & 0.08 & 0.133 \\
\hline Water & 55 & 55 & 0 & 0 \\
\hline Total Cost & & & & 0.1534 \\
\hline $\begin{array}{l}\text { Lt-nMn } \\
\text { adsorbent }\end{array}$ & 1.6 & --- & & \\
\hline $\begin{array}{l}\text { Cost of Lt-nMn } \\
\text { adsorbent } \\
\text { (USD/g) }\end{array}$ & \multicolumn{5}{|l}{} \\
\hline
\end{tabular}




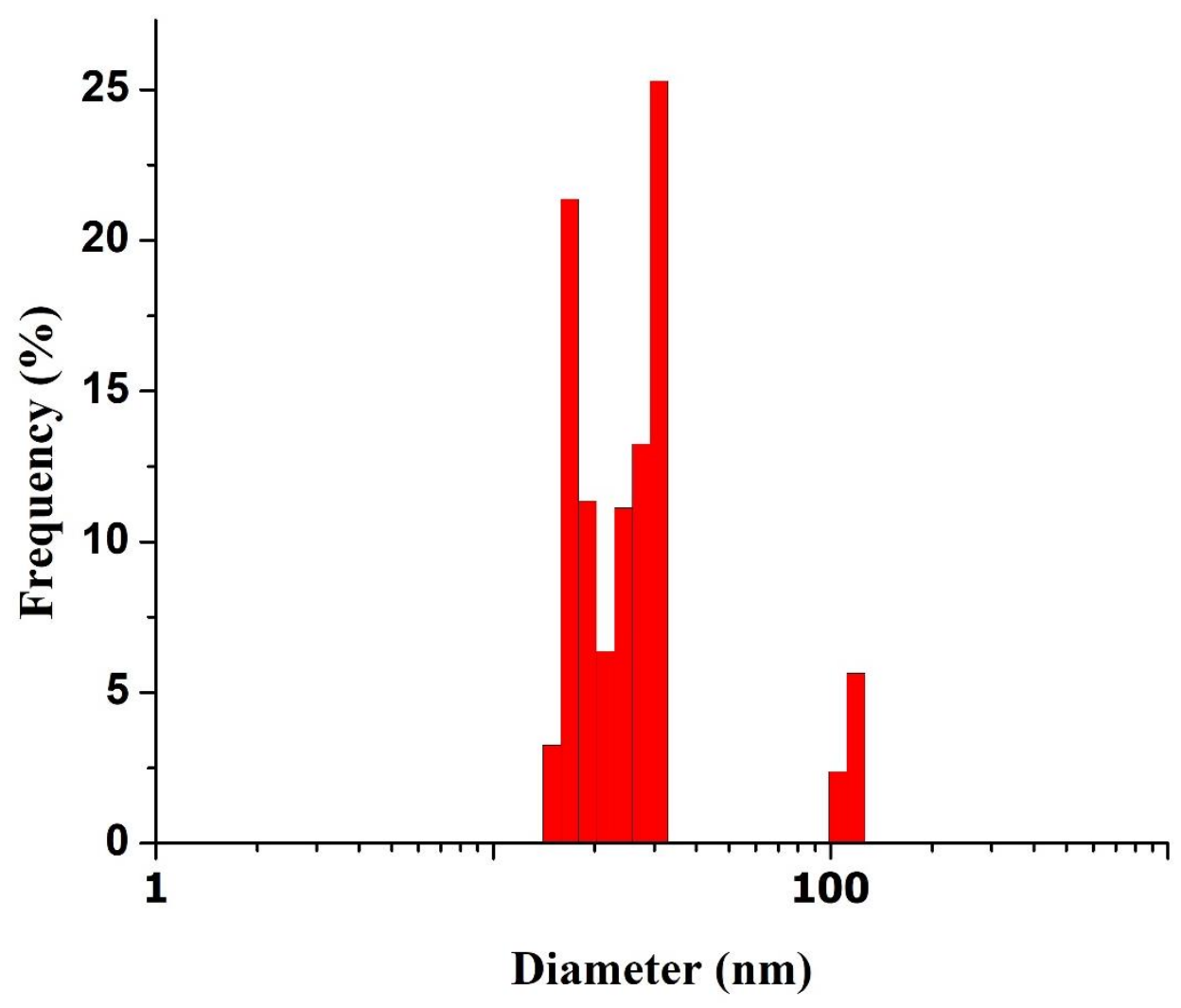

Fig. 1: Dynamic light scattering (DLS) analysis of Mn nanoparticles 


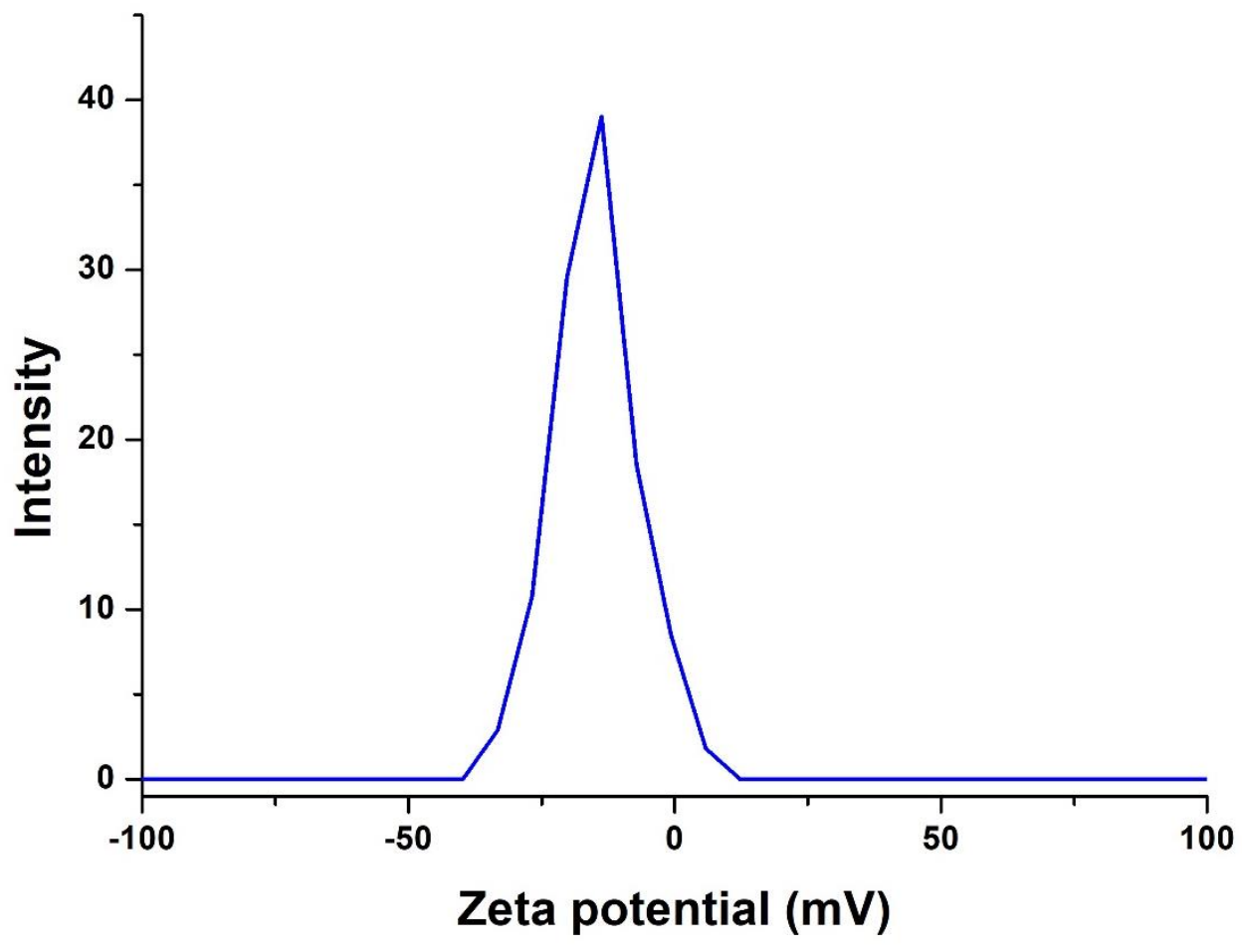

Fig. 2: Zeta potential analysis of Mn nanoparticles 


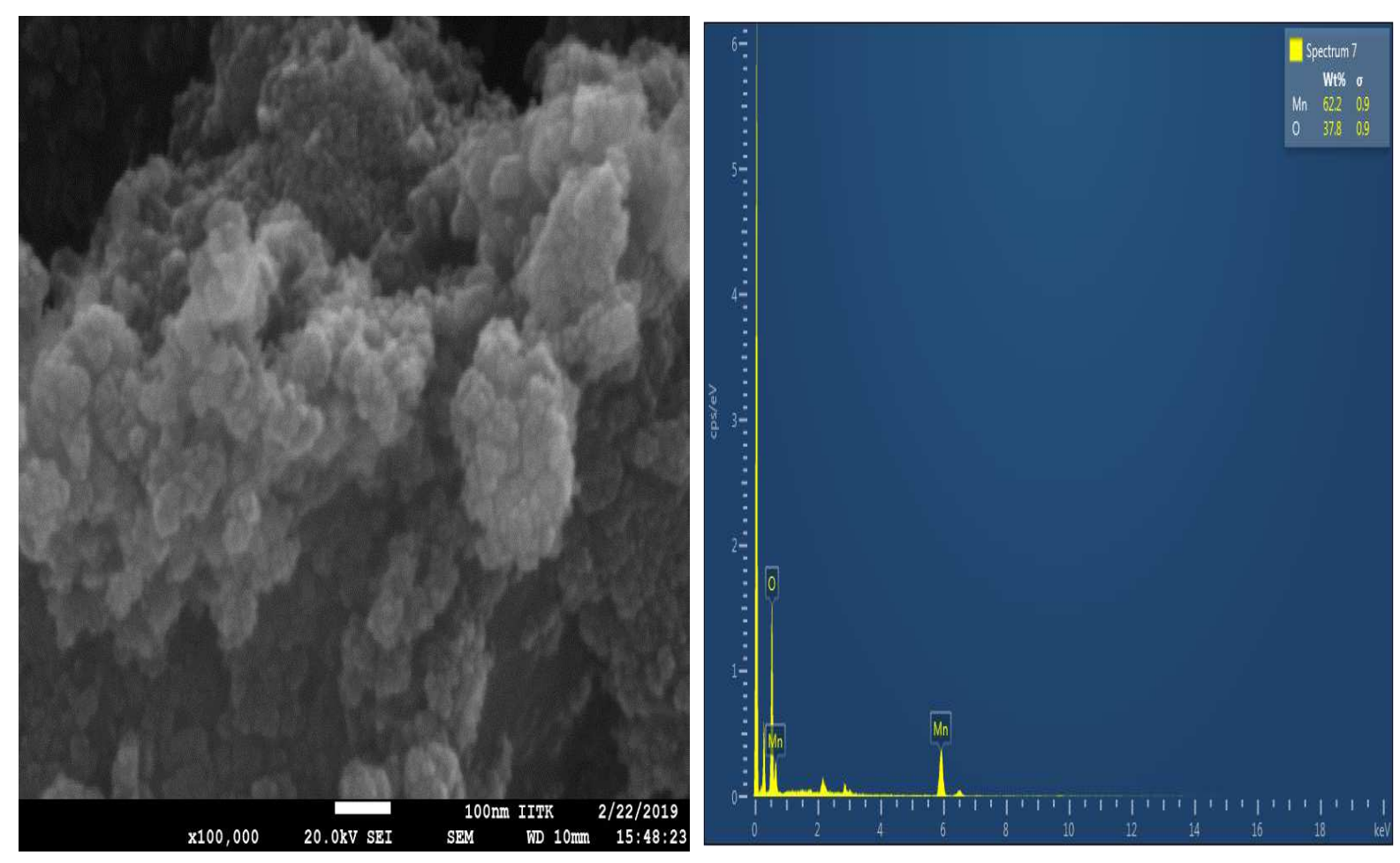

Fig. 3: FESEM and EDX analysis of Mn nanoparticles 

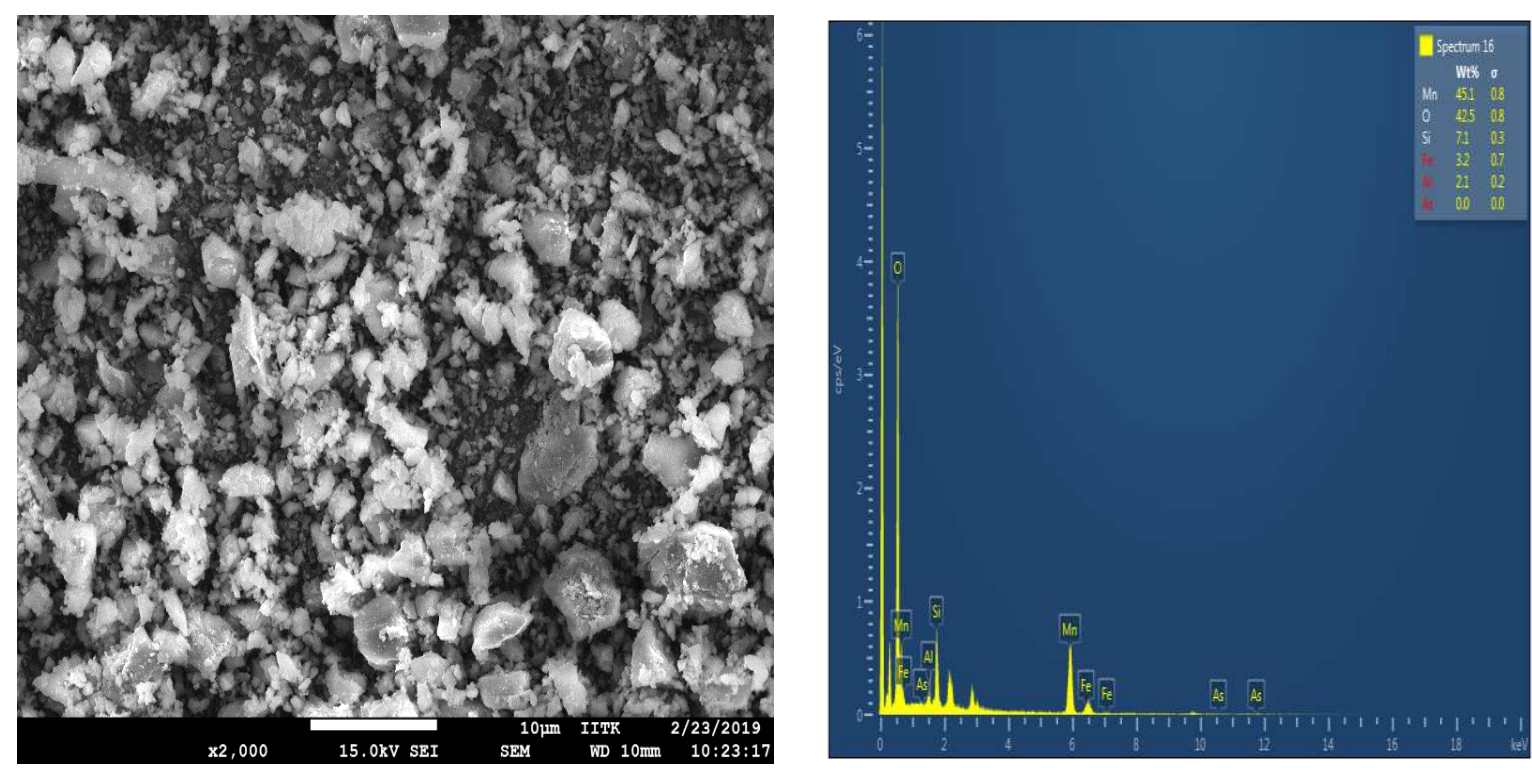

Fig. 4: FESEM and EDX analysis of Lt-nMn adsorbent particles 


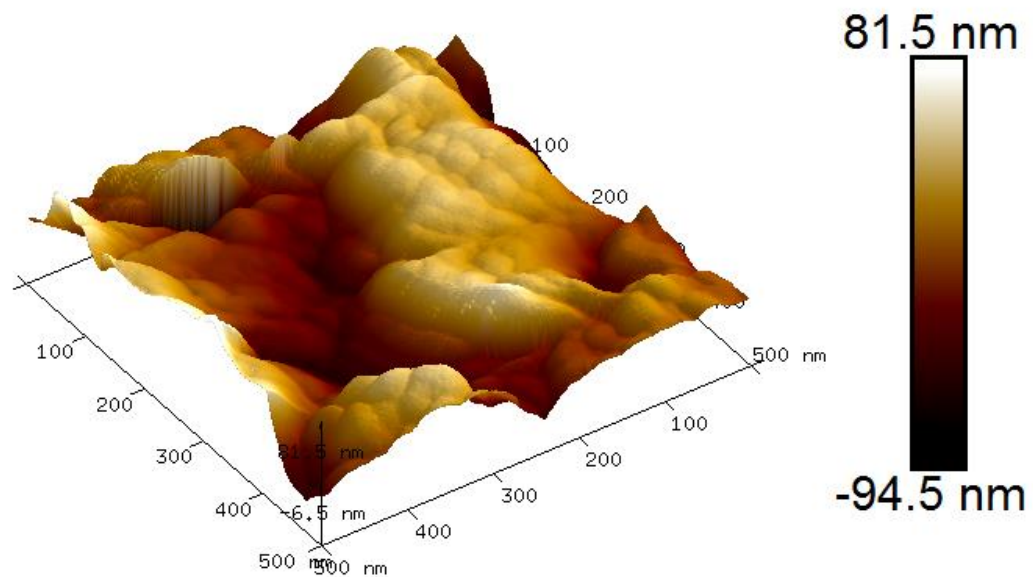

Height Sensor

$$
\overline{00.0 \mathrm{~nm}}
$$

Fig. 5: AFM analysis of Mn nanoparticles 


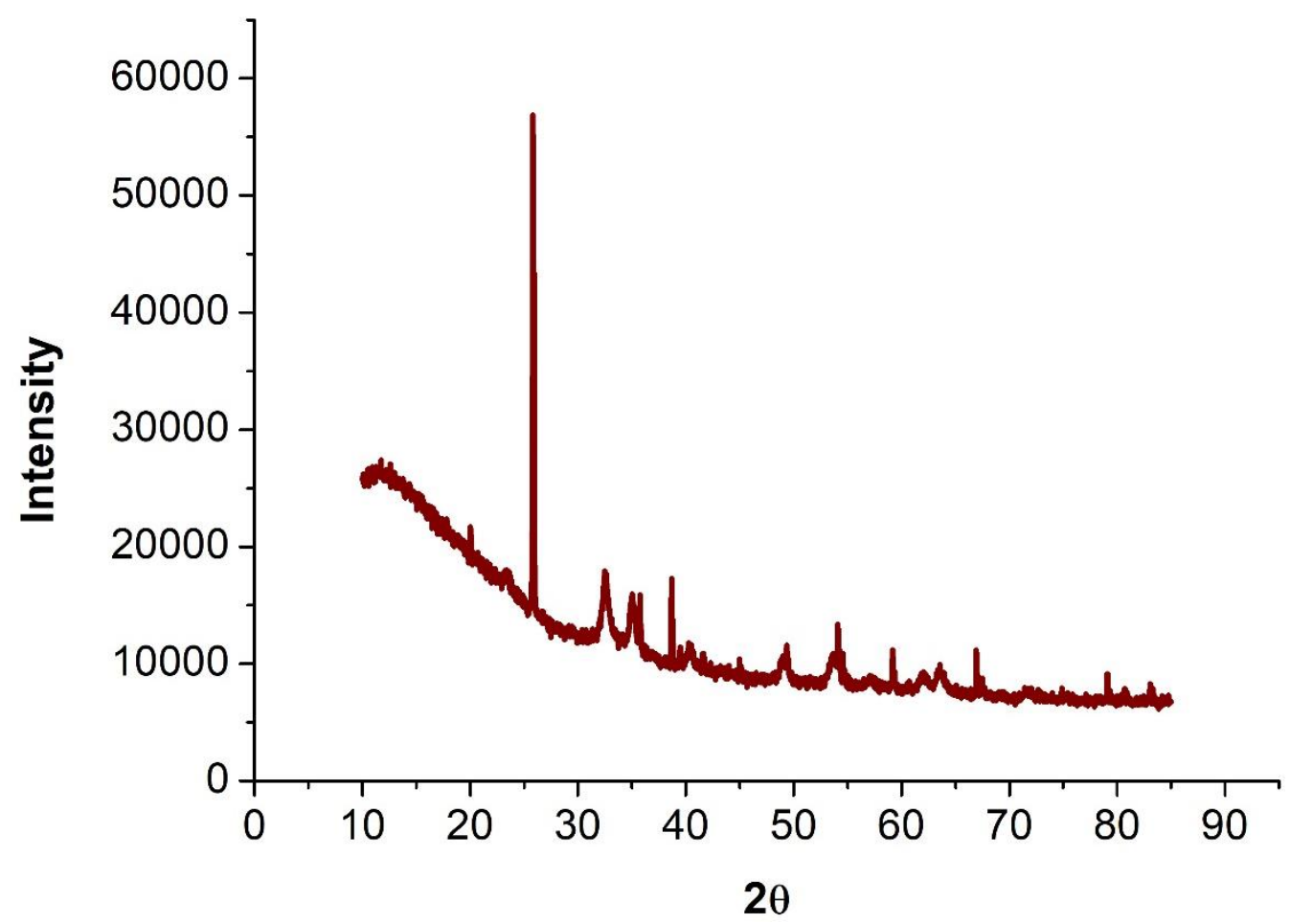

Fig. 6: XRD pattern of Lt-nMn adsorbent particles 


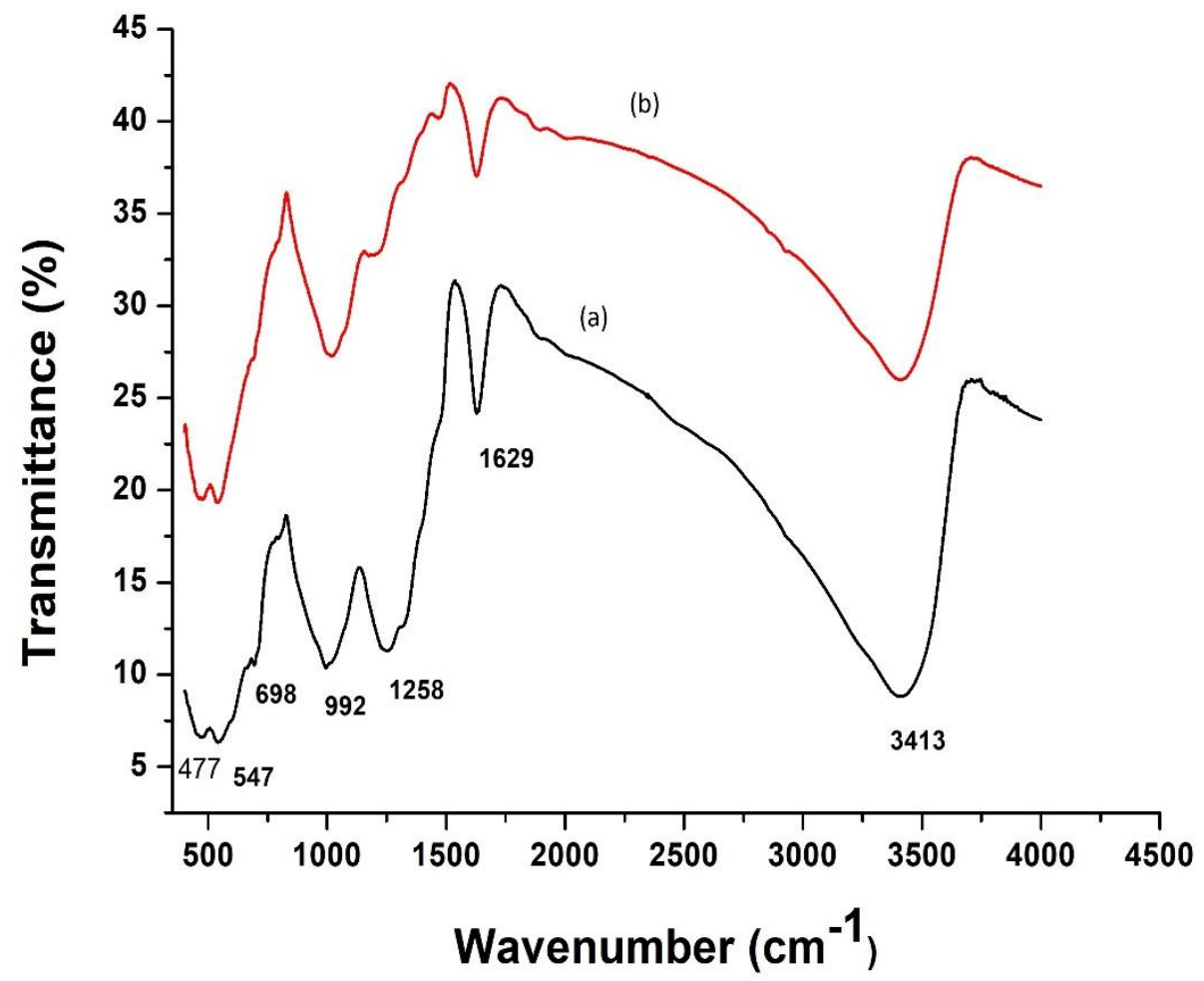

Fig. 7: FTIR spectra of synthesized Lt-nMn particles (a) before adsorption and (b) after adsorption of As(III) 


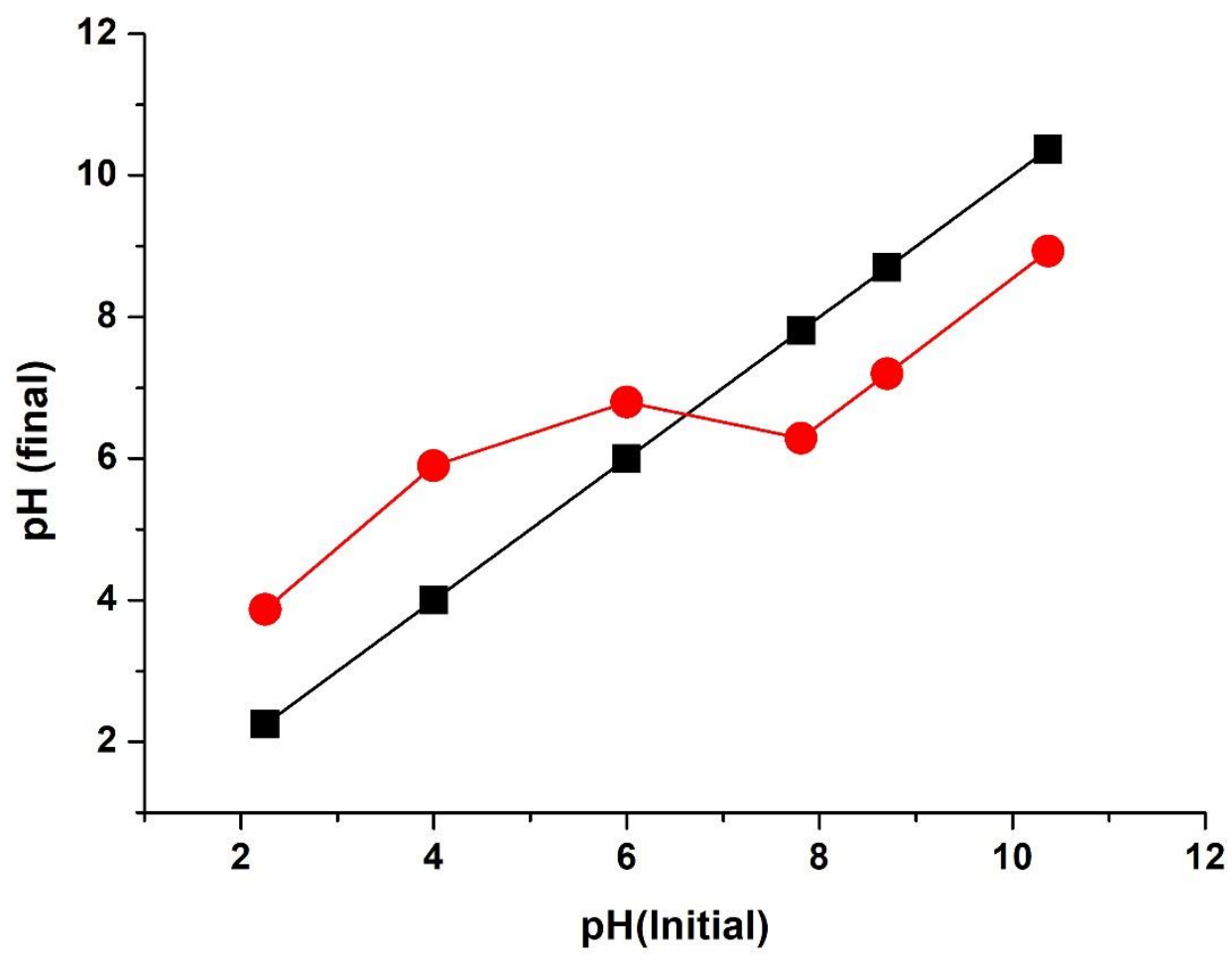

Fig. 8: Zero point charge (pHzPc) of Lt-nMn adsorbent particles 


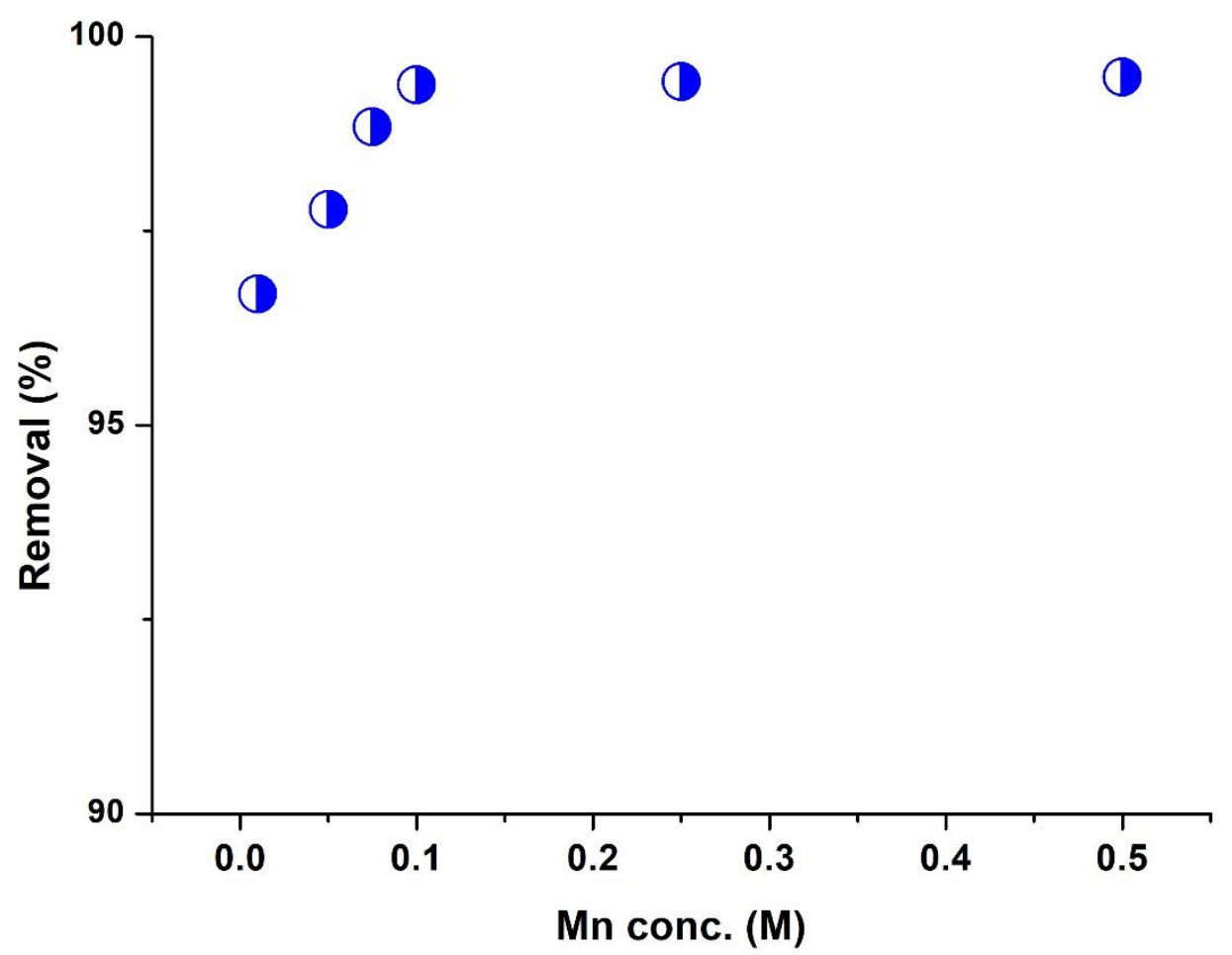

Fig. 9: Effect of nMn loading on As(III) removal 


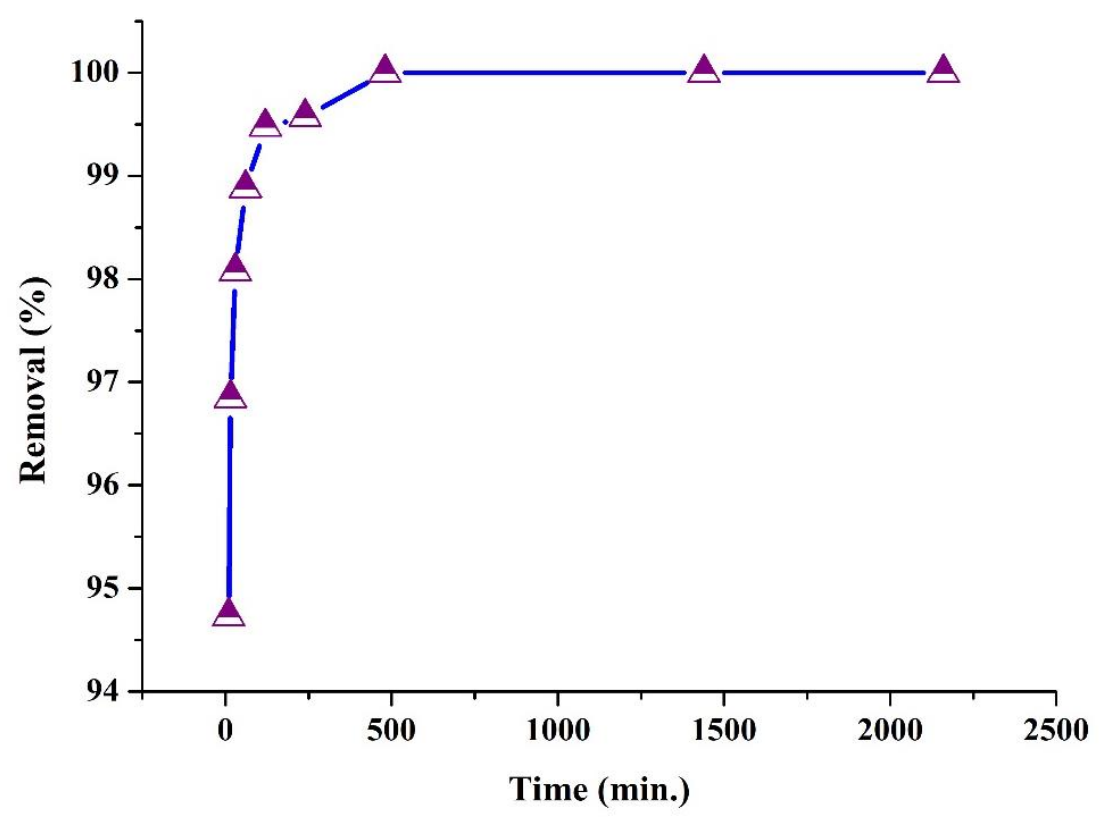

Fig. 10: Effect of contact time on As(III) removal (experimental conditions: i.c - 100 ppb, dose $-1 \mathrm{~g} / \mathrm{L}, \mathrm{pH}-7 \pm 0.2$, temp: $298 \mathrm{~K}$, contact time: 10 - $2160 \mathrm{~min}$, stirring speed - 150 rpm) 


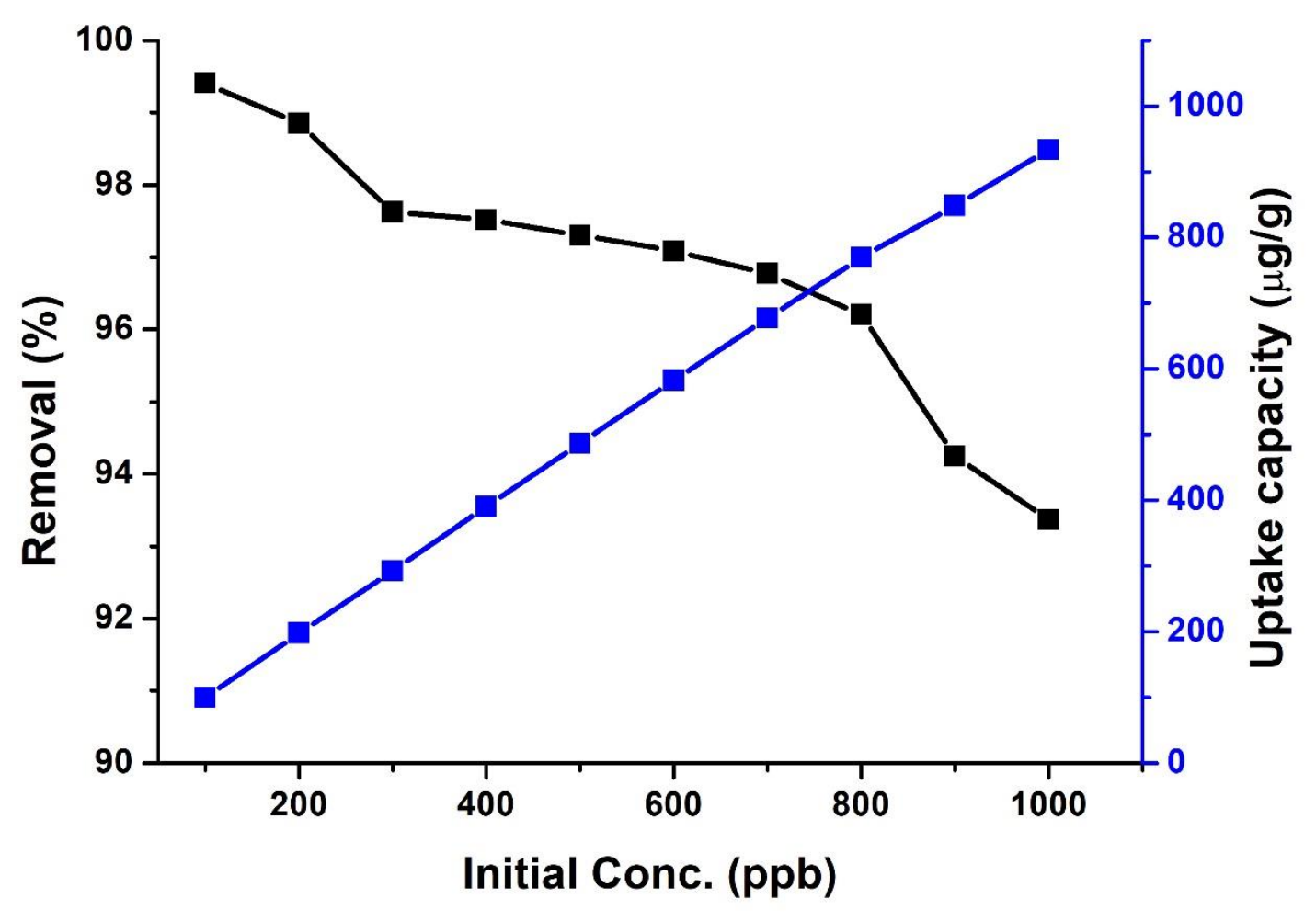

Fig. 11: Effect of initial concentration on $\mathrm{As}(\mathrm{III})$ removal [experimental conditions: i.c -

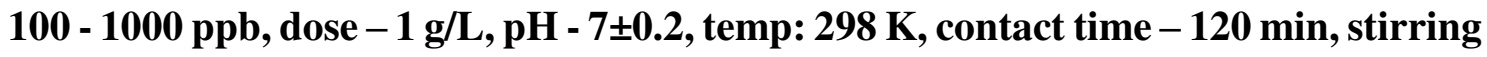
speed - $150 \mathrm{rpm}$ ] 


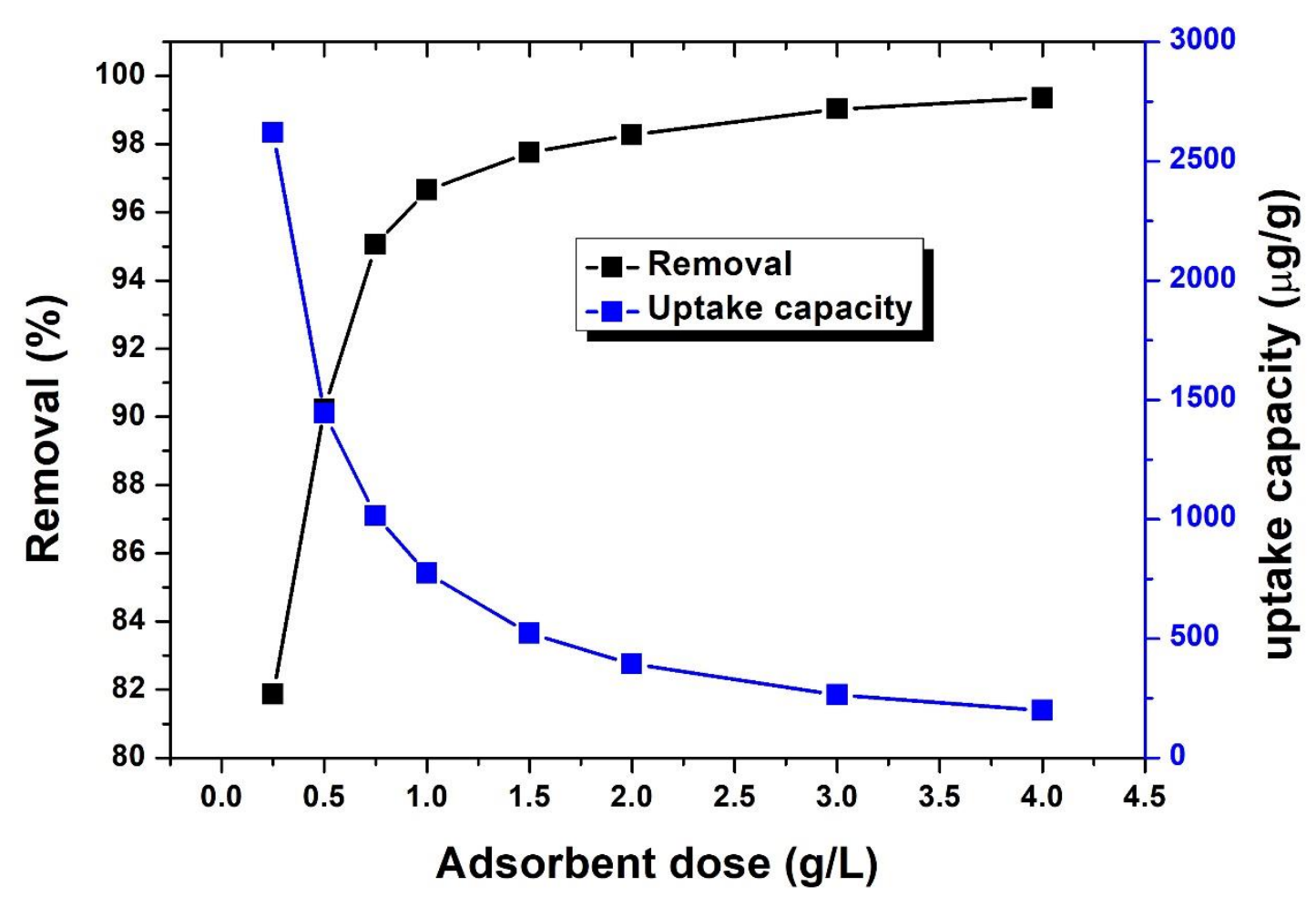

Fig. 12: Effect of adsorbent dose on As(III) removal [experimental conditions: i.c - 800 ppb As (III), dose: $0.25-4 \mathrm{~g} / \mathrm{L}$, pH - 7士0.2, temp: $298 \mathrm{~K}$, contact time $-120 \mathrm{~min}$, stirring speed - $150 \mathrm{rpm}]$ 


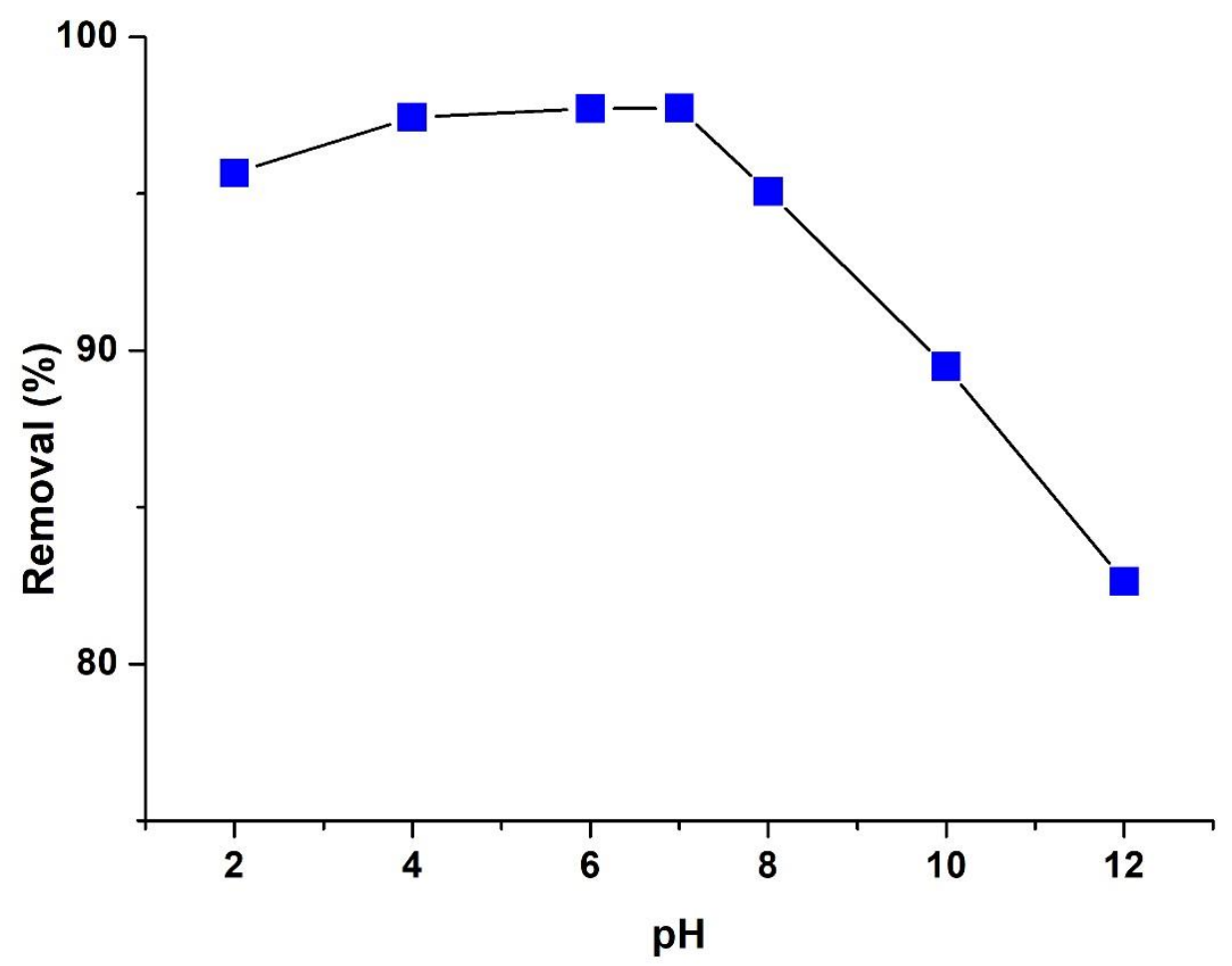

Fig. 13: Effect of pH on As(III) removal [experimental conditions: i.c - $800 \mathrm{ppb} \mathrm{A}$, dose $1.5 \mathrm{~g} / \mathrm{L}, \mathrm{pH}-2-12$, temp: $298 \mathrm{~K}$, contact time $-120 \mathrm{~min}$, stirring speed $-150 \mathrm{rpm}]$ 


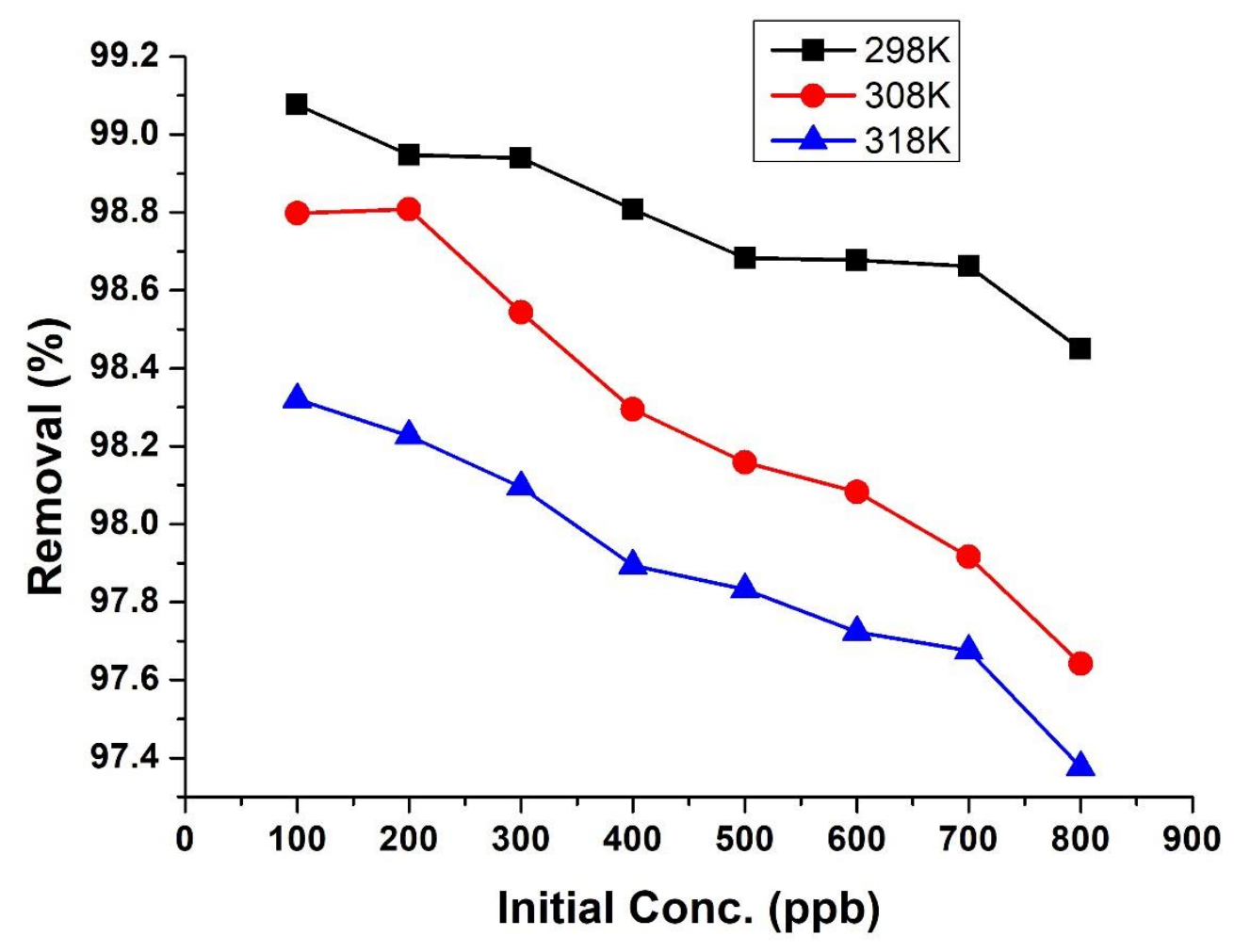

Fig. 14: Effect of temperature on As(III) removal (experimental conditions: i.c - 100-800 ppb, dose $-1.5 \mathrm{~g} / \mathrm{L}, \mathrm{pH}-7 \pm 0.2$, temp: 298 - $318 \mathrm{~K}$, contact time $-120 \mathrm{~min}$, stirring speed - 150 rpm) 


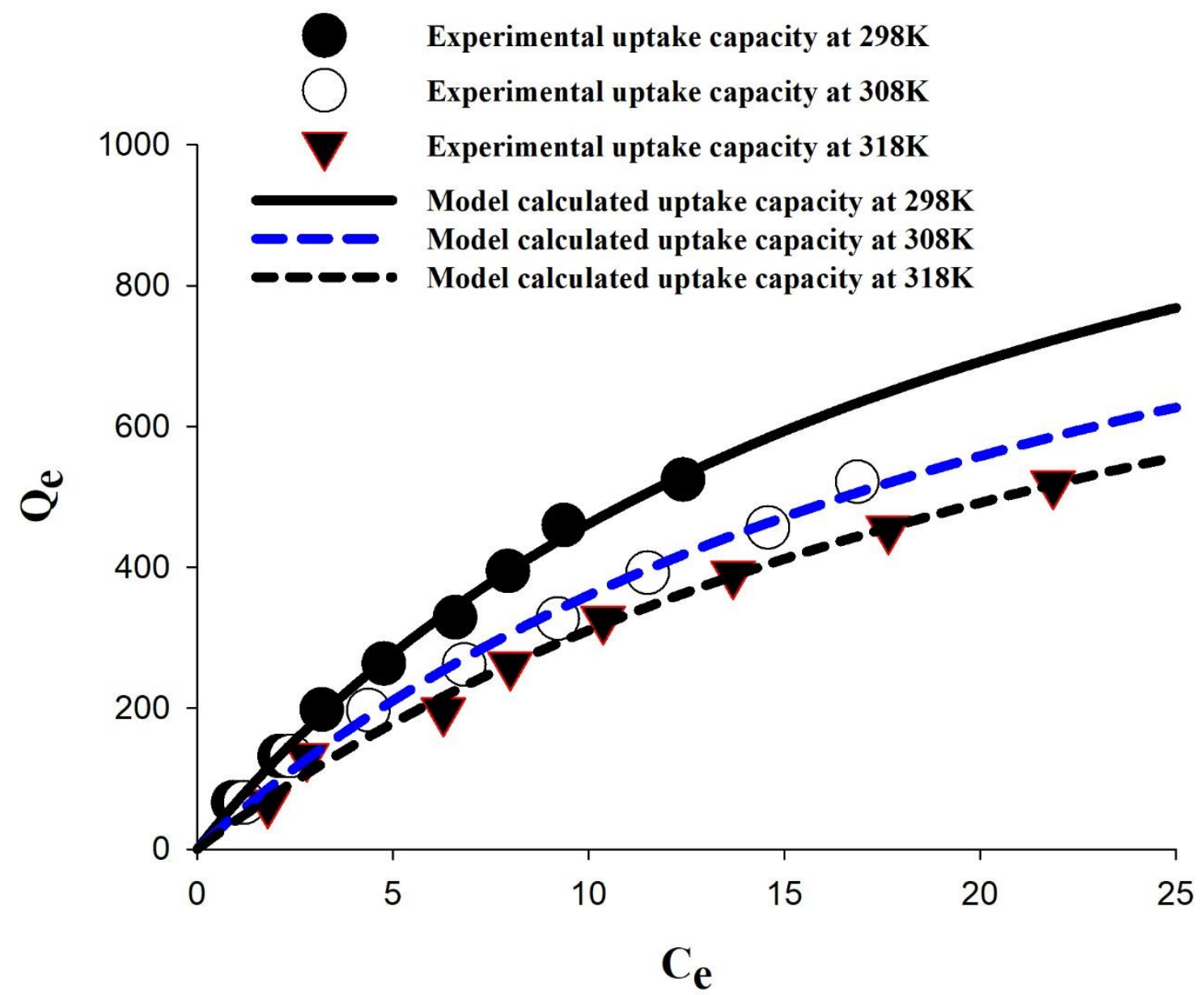

Fig. 15: Langmuir isotherm model for As(III) adsorption (experimental conditions: i.c 100- 800 ppb, dose - $1.5 \mathrm{~g} / \mathrm{L}, \mathrm{pH}-7 \pm 0.2$, temp. - 298 - $318 \mathrm{~K}$, contact time - 120 min, stirring speed - 150 rpm) 


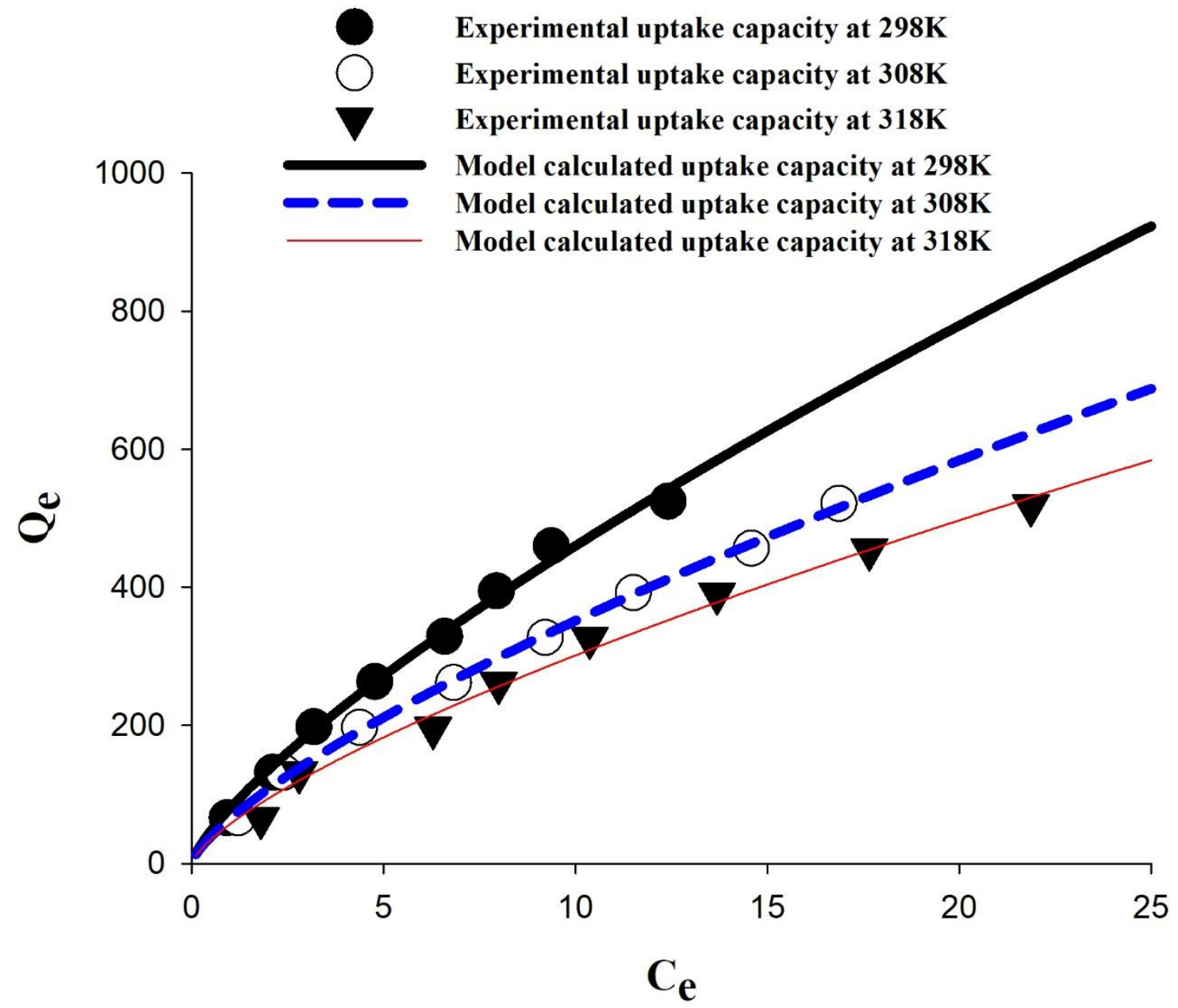

Fig. 16: Freundlich isotherm model for As(III) adsorption (experimental conditions: i.c 100- $800 \mathrm{ppb}$, dose - $1.5 \mathrm{~g} / \mathrm{L}, \mathrm{pH}-7 \pm 0.2$, temp. - 298 - $318 \mathrm{~K}$, contact time - 120 min, stirring speed - 150 rpm) 


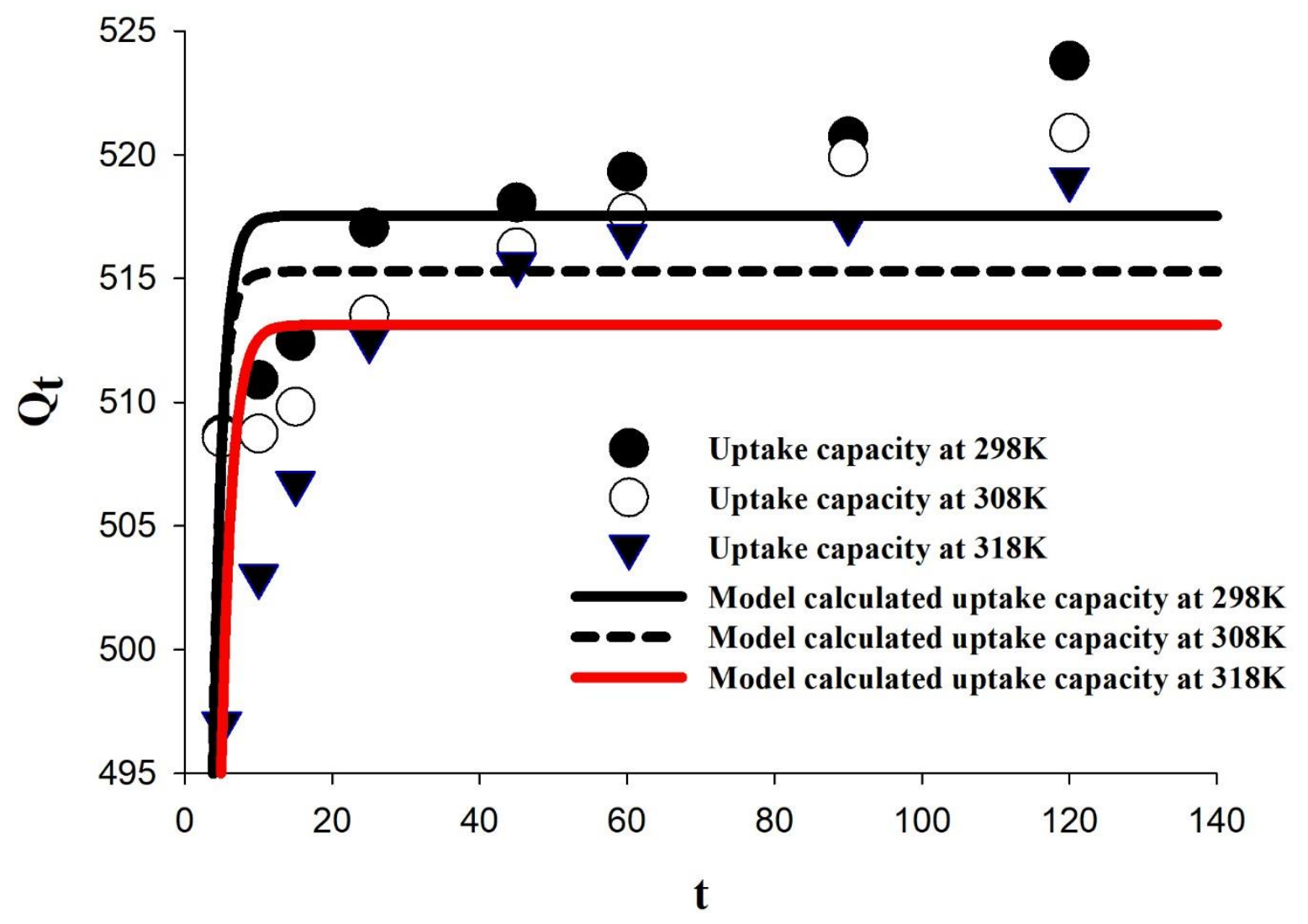

Fig. 17: Pseudo - first - order kinetic model (experimental conditions: i.c - 800 ppb, dose $-1.5 \mathrm{~g} / \mathrm{L}, \mathrm{pH}-7 \pm 0.2$, temp: $298-318 \mathrm{~K}$, contact time -5 - $120 \mathrm{~min}$, stirring speed - 150 rpm) 


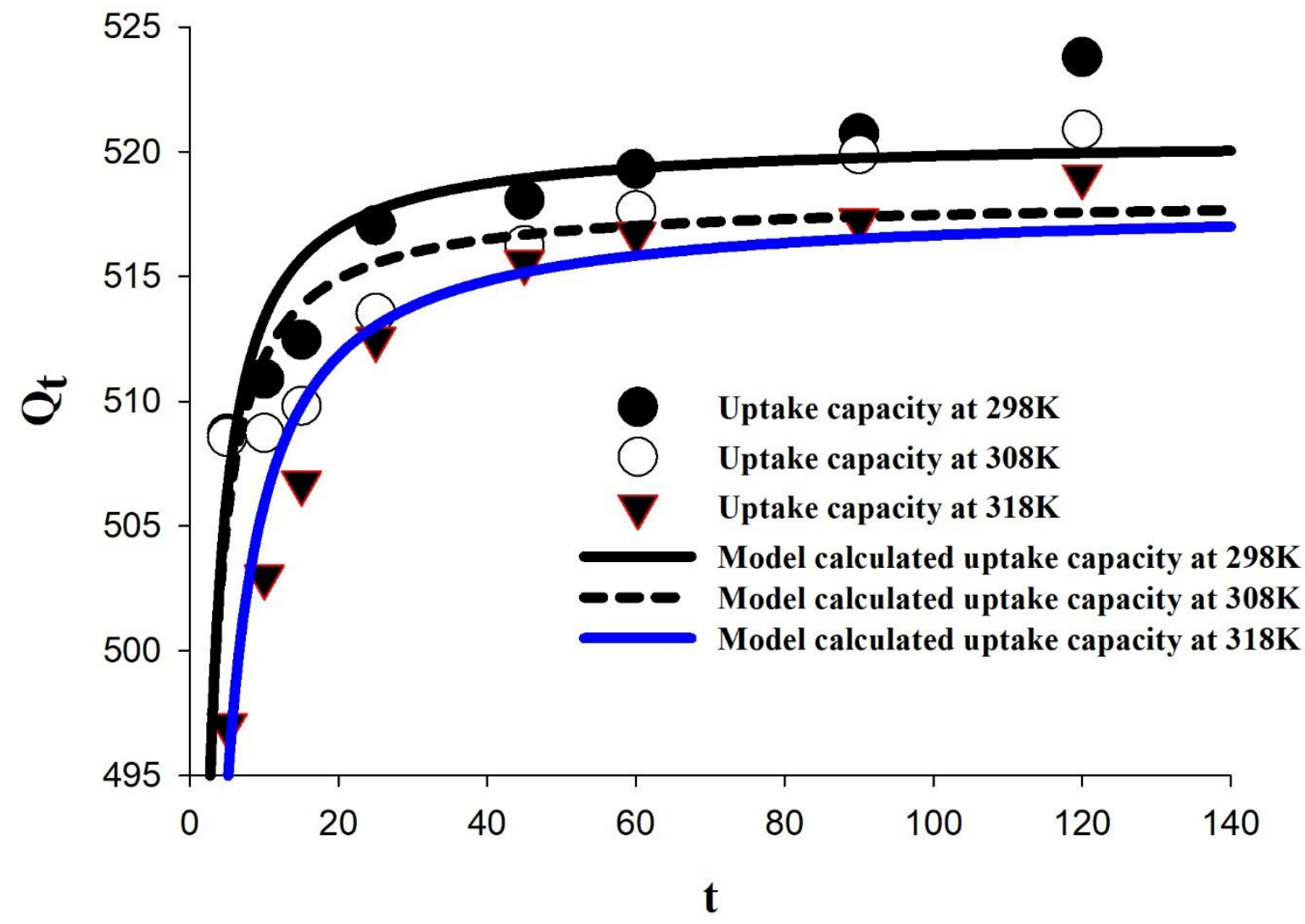

Fig. 18: Pseudo - second - order kinetic model (experimental conditions: i.c - 800 ppb,

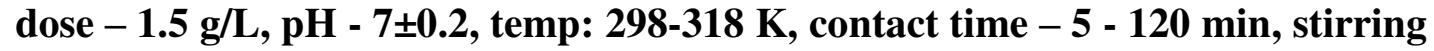
speed - 150 rpm) 


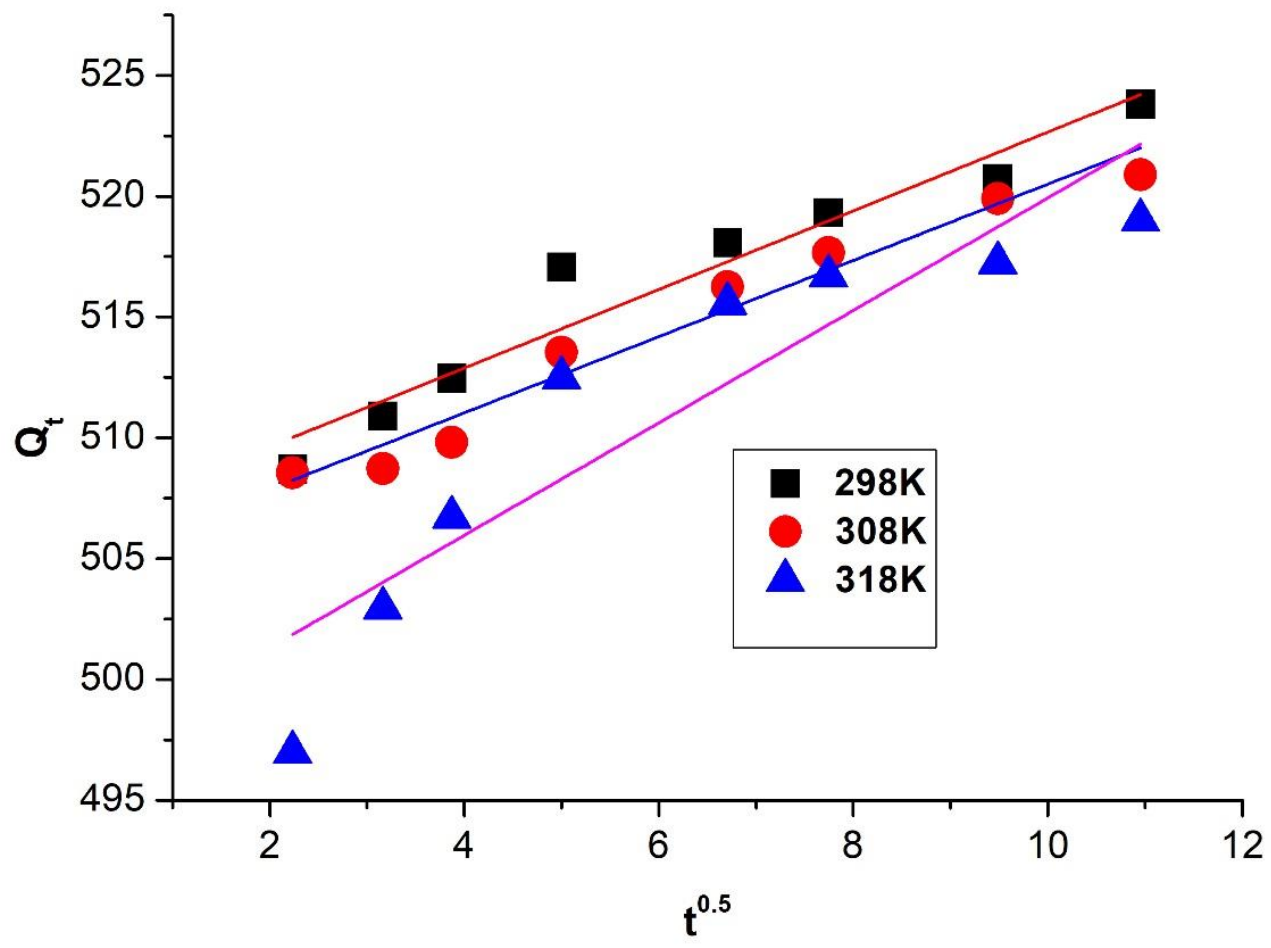

Fig. 19: Intra particle diffusion kinetic model (experimental conditions: i.c - 800 ppb, dose $-1.5 \mathrm{~g} / \mathrm{L}, \mathrm{pH}-7 \pm 0.2$, temp: 298-318 K, contact time - 5 - $120 \mathrm{~min}$, stirring speed - 150 rpm) 


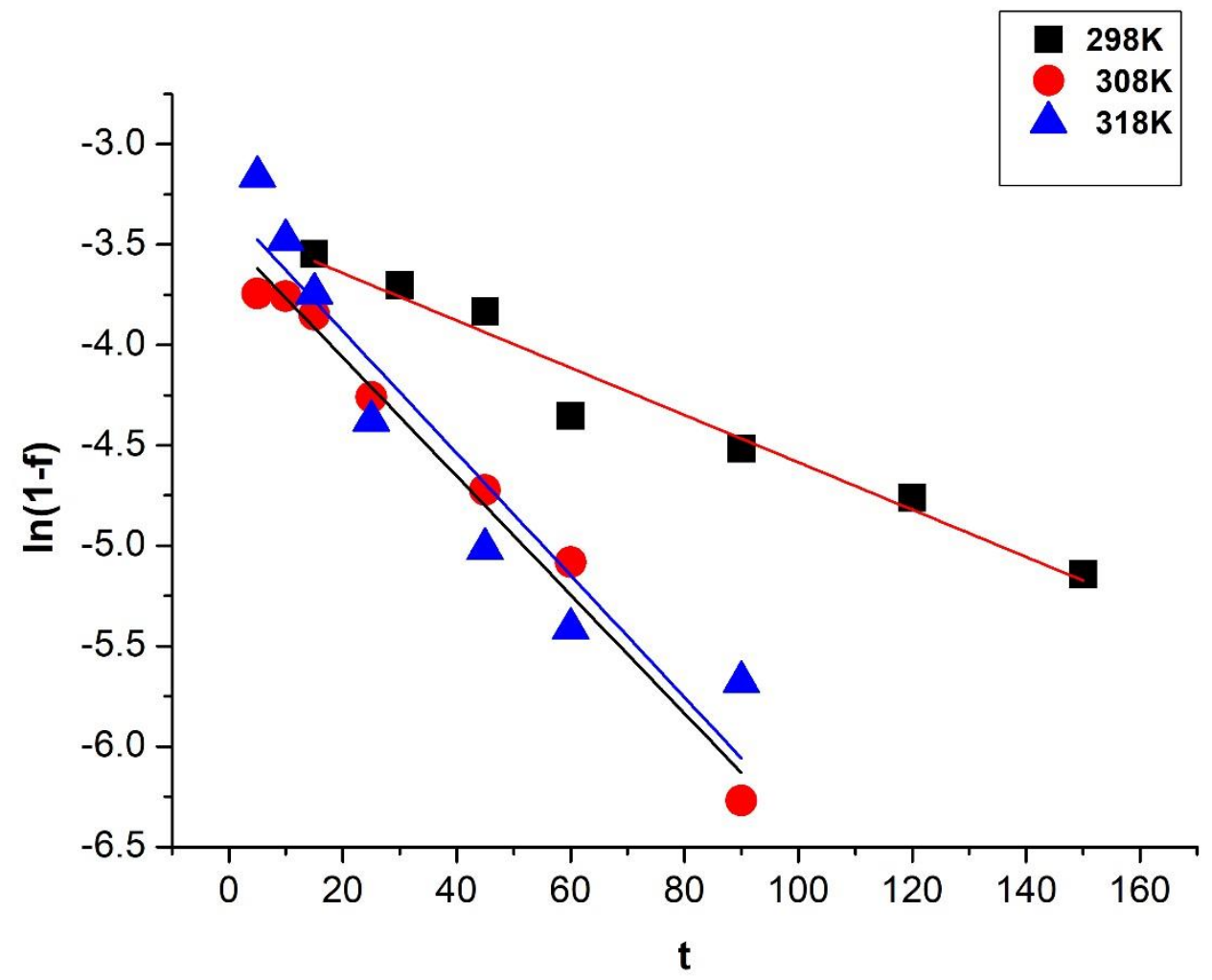

Fig. 20: Liquid film kinetic model (experimental conditions: i.c $-800 \mathrm{ppb}$, dose -1.5 $\mathrm{g} / \mathrm{L}, \mathrm{pH}$ - 7士0.2, temp: 298-318 K, contact time - 5 - $120 \mathrm{~min}$, stirring speed - $150 \mathrm{rpm}$ ) 


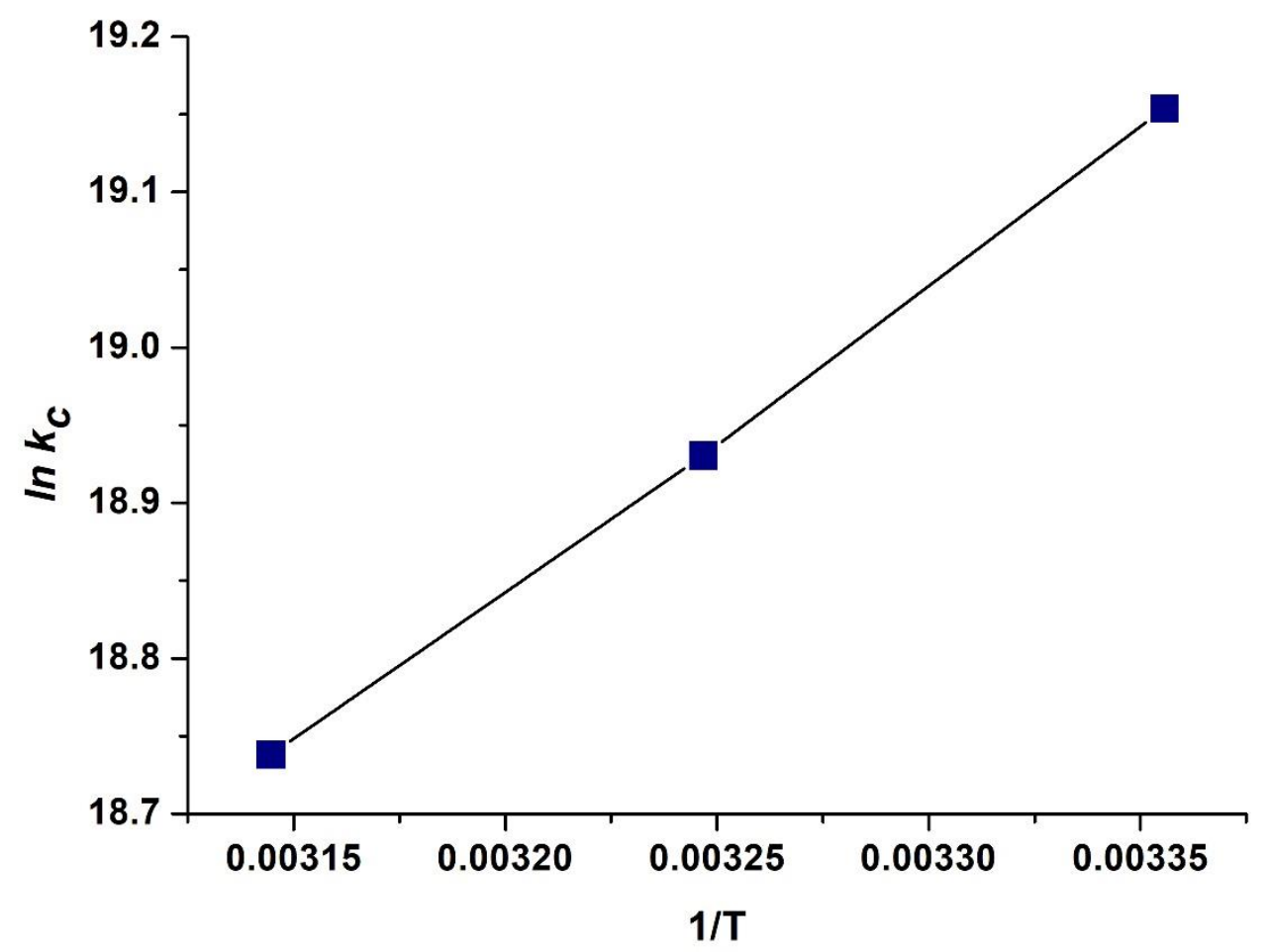

Fig. 21: Thermodynamic studies for determination of Gibb's free energy $\left(\Delta G^{\circ}\right)$, change of standard enthalpy $\left(\Delta \mathrm{H}^{\circ}\right)$ and change of standard entropy $\left(\Delta \mathrm{S}^{\circ}\right)$ for $A s$ (III) removal 


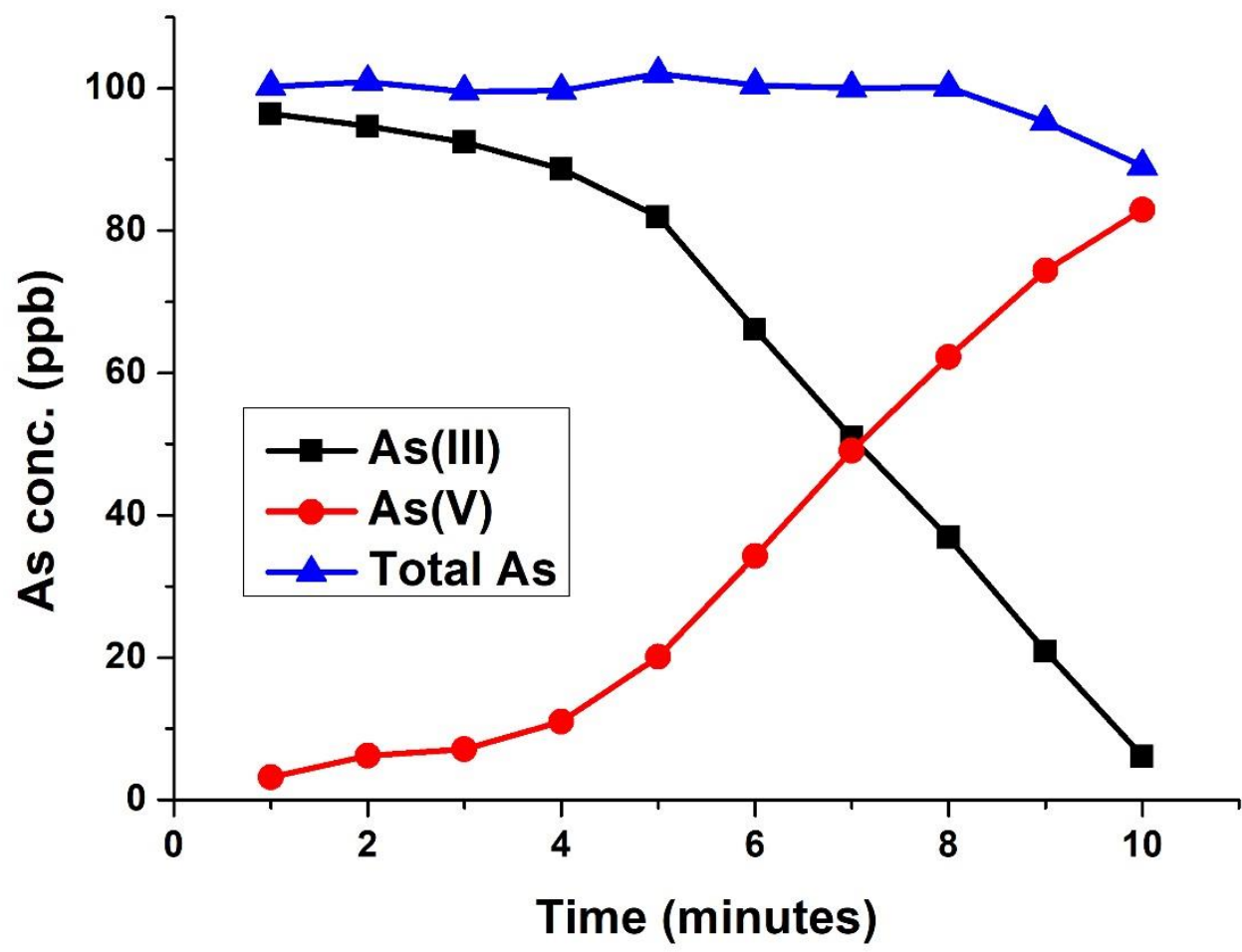

Fig. 22: Oxidation of $\mathrm{As}(\mathrm{III})$ to $\mathrm{As}(\mathrm{V})$ during arsenic removal using Lt-nMn adsorbent (experimental conditions: i.c $=100 \mathrm{ppb}$ As(III); dose $=1.5 \mathrm{~g} / \mathrm{L} ; \mathrm{pH}=7 \pm 0.2$; Temperature $=298 \mathrm{~K}$ ) 


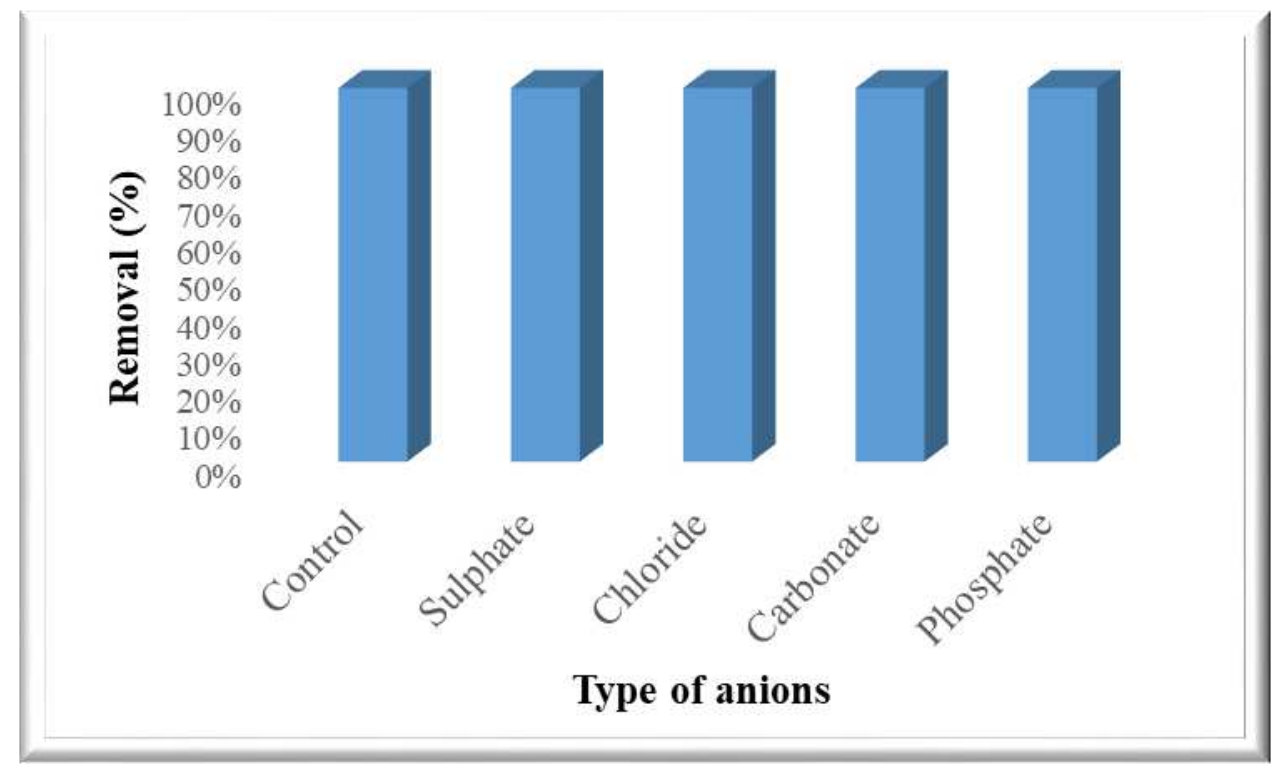

Fig. 23: Effect of competing anions on As(III) adsorption (Experimental conditions: i.c $800 \mathrm{ppb}$, competing ions conc. $-2000 \mathrm{ppb}$, adsorbent dose $-1.5 \mathrm{~g} / \mathrm{L}, \mathrm{pH}-7 \pm 0.2$, temp. - $298 \mathrm{~K}$, stirring speed - $150 \mathrm{rpm}$ ) 


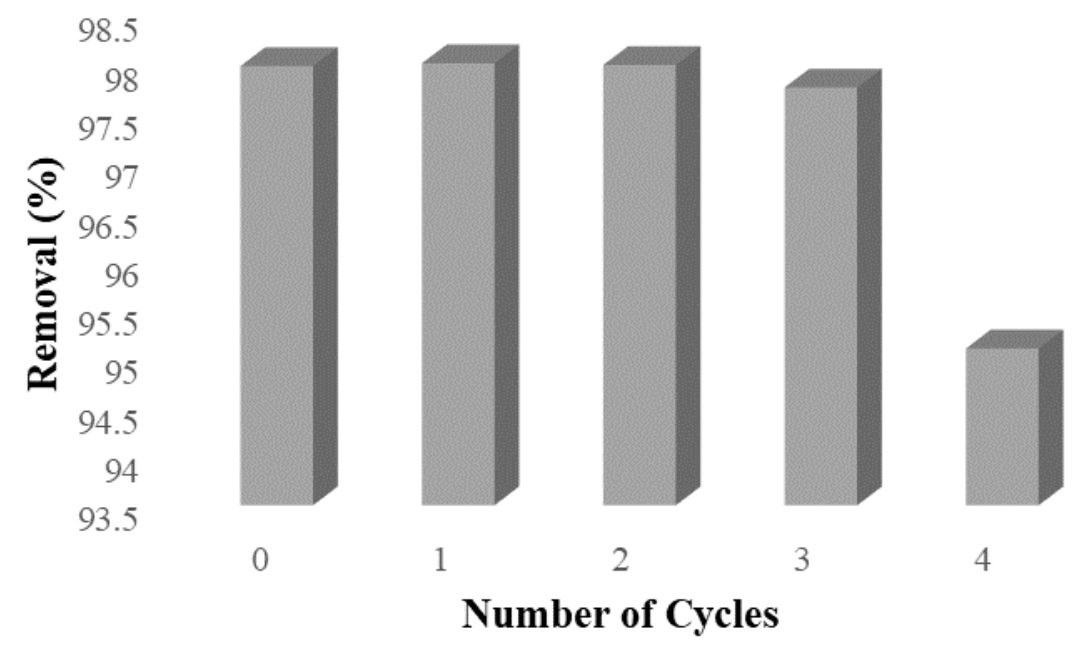

Fig. 24: Regeneration study of Lt-nMn adsorbent (Experimental conditions: i.c of As (III) - $800 \mathrm{ppb}$, normality of $\mathrm{NaOH}-0.1 \mathrm{~N}$, adsorbent dose $-1.5 \mathrm{~g} / \mathrm{L}$, contact time - 120 min, stirring spped - 150 rpm) 


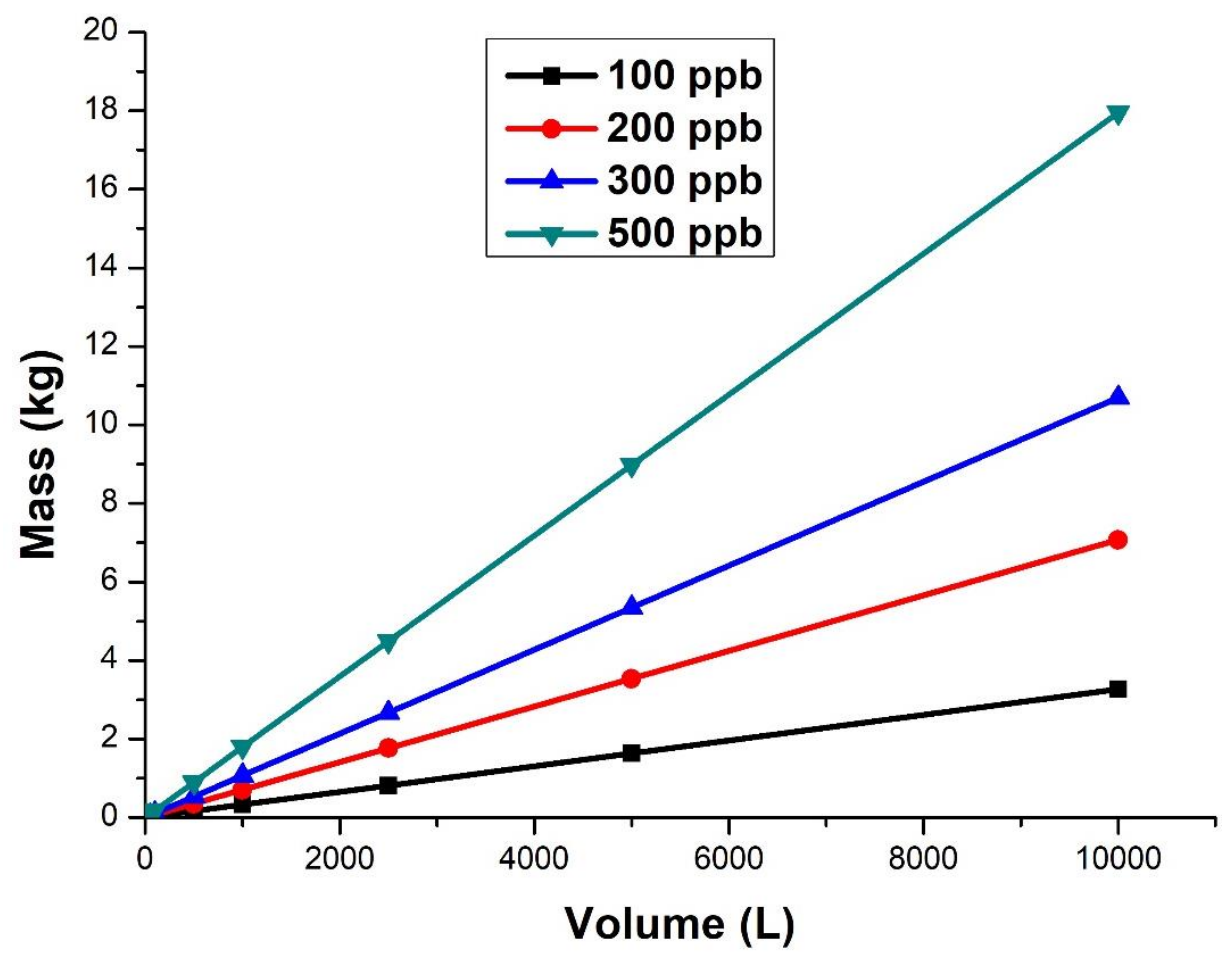

Fig. 25: Amount of Lt-nMn required for treating different volume with different arsenic concentrations 